\title{
Multifunctional Mesoporous Polydopamine With Hydrophobic Paclitaxel For Photoacoustic Imaging-Guided Chemo-Photothermal Synergistic Therapy
}

This article was published in the following Dove Press journal:

International Journal of Nanomedicine

\section{Liren Zhang \\ Peng Yang \\ Ranran Guo \\ Jiaxin Sun \\ Ruihong Xie \\ Wuli Yang (D)}

State Key Laboratory of Molecular Engineering of Polymers, Department of Macromolecular Science, Fudan University, Shanghai 200433, People's Republic of China
Correspondence: Wuli Yang

State Key Laboratory of Molecular

Engineering of Polymers, Department of

Macromolecular Science, Fudan

University, Shanghai 200433, People's

Republic of China

Tel +86-02 I-3 I 242385

Email wlyang@fudan.edu.cn
Background: Chemo-photothermal therapy has attracted intensive attention because of its low side effects and better therapeutic efficiency. Although many photothermal agents have been loaded with chemotherapeutic drugs for chemo-photothermal therapy, their applications are limited by complex synthetic protocols and long-term safety. Therefore, there is significant clinical value in the development of a simple system of biocompatible and biodegradable photothermal nanomaterials with high payloads of chemotherapeutic drugs for chemo-photothermal synergistic therapy.

Materials and methods: In this study, PEG-modified polydopamine nanoparticles with mesoporous structure (MPDA-PEG) were successfully obtained by an emulsion-induced interface assembly strategy. Subsequently, paclitaxel (PTX) dissolved in acetone was loaded into the mesoporous channels of MPDA-PEG nanoparticles by solution absorption method. A PTX-loaded MPDA-PEG (MPDA-PEG-PTX) nanoplatform for combination of photothermal therapy (PTT) and chemotherapy was developed.

Results: The synthesized MPDA-PEG nanoparticles had a great photothermal effect under nearinfrared (NIR) laser irradiation and exhibited an enhanced photothermal effect with the increase of particle size. Meanwhile, MPDA-PEG nanoparticles also had a high payload of PTX, and the PTX release could be greatly accelerated by elevated temperature from photothermal effect. In MTT cytotoxicity assay, A549 cells incubated with MPDA-PEG-PTX under NIR laser irradiation (PTT + chemotherapy group) exhibited better therapeutic effect than single chemotherapy (MPDA-PEGPTX group) and PTT (MPDA-PEG + Laser group). The synergistic therapeutic effect of MPDAPEG-PTX with NIR laser irradiation in vivo was further investigated under the guidance of photoacoustic imaging (PAI), tumors of nude mice treated with MPDA-PEG-PTX with NIR laser irradiation were completely eliminated with minimal side effect.

Conclusion: The MPDA-PEG-PTX nanoplatform is a simple and effective platform which can completely inhibit tumor growth with minimal side effects under NIR irradiation, and it exhibits better therapeutic effect than single chemotherapy and PTT.

Keywords: photothermal therapy, polydopamine, mesoporous nanoparticle, chemotherapy, paclitaxel

\section{Introduction}

Cancer has been one of the major threats to the health of human beings for many years. ${ }^{1}$ At present, chemotherapy is one of the most common anticancer strategy in clinical. ${ }^{2}$ However, conventional chemotherapy is suffering from great limitation due to low 
therapeutic outcomes, serious side effects and poor druggability as many chemotherapeutic molecules cannot be dissolved in aqueous solution. ${ }^{3-5}$ Recently, many cooperative therapeutic methods, which combined chemotherapy with photothermal therapy (PTT), photodynamic therapy (PDT), immunotherapy and gene therapy have been developed to overcome the limitations of chemotherapy. ${ }^{6-9}$ Above all, photothermal therapy is an attractive way because of its minimal invasion, easy applicability, high efficiency and low systemic toxicity. ${ }^{10,11}$ In particularly, chemo-photothermal therapy has been proved to be an useful strategy for cancer treatment in a synergistic manner as the elevated temperature can facilitate the release of loaded drugs in tumor site and increase the sensitivity of tumor to chemotherapeutic drugs. ${ }^{12}$ In recent years, some photothermal agents, including gold nanostructures, carbon nanomaterials and $\mathrm{CuS}$ nanoparticles, have been loaded with chemotherapeutic drugs for chemophotothermal therapy. ${ }^{13-17}$ Although these systems exhibit some advantages, their applications are limited by complex synthetic protocols and long-term safety. ${ }^{18,19}$ Therefore, a simple system of biocompatible and biodegradable photothermal nanomaterialwith high payloads for chemotherapeutic drugs is needed to enlarge the clinical application of chemophotothermal synergistic therapy.

As possessing the advantages of facile synthesis and easy modification, polydopamine (PDA), a nature-inspired melanin-like synthetic polymer material, has attracted growing attention in various applications across the chemical, biological, medical and materials sciences fields. ${ }^{20-23}$ Polydopamine materials also have excellent biocompatibility and biodegradability basically not induce long-term toxicity in vivo. ${ }^{24}$ Recently, PDA has emerged as a photothermal therapeutic agent for in vivo cancer therapy as its strong near-infrared (NIR) absorption and high photothermal conversion efficiency. ${ }^{25}$ Most especially, the existence of massive aromatic rings in PDA nanoparticles can lead to $\pi-\pi$ stacking with chemotherapeutic drugs, ${ }^{26,27}$ however, the anticancer drug-loading content (DLC) of typical PDA materials is still subject to the limited external surfaces. Noteworthy mesoporous structure would be favorable to improve its DLC, these attractive characteristics made PDA material with mesoporous structure a candidate for combined PTT and chemotherapy.

Herein, PEG-modified mesoporous polydopamine (MPDA-PEG) with large pore volume is successfully obtained for chemo-photothermal synergistic therapy of tumor. The fabrication of MPDA-PEG nanoparticles is elucidated in Scheme 1. As prepared MPDA-PEG nanoparticles have an outstanding photothermal conversion efficiency $(\sim 41 \%)$ and an enhanced phototherapy effect with the increase of particle size. Moreover, they also have a high payload of paclitaxel (PTX, DLC $=15 \%$ ), and PTX release activity could be accelerated by elevated temperature greatly. Subsequently, the therapeutic effect of PTX-loaded MPDA-PEG is investigated on a small animal model, where tumors are entirely eliminated with minimal systemic toxicity and side effects, demonstrating better therapeutic efficacy than single PTT or chemotherapy. More importantly, MPDA-PEG nanoparticles own a high-resolution photoacoustic signal in vitro and in vivo. Therefore, PTX-loaded MPDAPEG could be used for chemo-photothermal synergistic therapy guiding by photoacoustic imaging (PAI).

\section{Materials And Methods Materials}

Dopamine hydrochloride, pluronic F127, remophor®EL $(\mathrm{pH}=6.0-8.0)$, paclitaxel and 3-(4,5-Dimethyl-2-thiazolyl)-2,5-diphenyl-2H-tetrazolium bromide (MTT) were obtained from Aladdin (Shanghai, People's Republic of China). Acetone, hydrogen peroxide $\left(\mathrm{H}_{2} \mathrm{O}_{2}, 30 \%\right)$, ethanol, 1,3,5-trimethylbenzene (TMB) and ammonia $\left(\mathrm{NH}_{3} \cdot \mathrm{H}_{2} \mathrm{O}, 25-28 \%\right)$ were purchased from Sinopharm Chemical Reagent Co. Ltd (Shanghai, People's Republic of China). Dimethyl sulfoxide was obtained from Shanghai Chemical Reagents Co. mPEG-NH $(M \mathrm{w}=4000)$ was bought from Pengsheng Biotechnology Co., Ltd. 4, 6-diamidino-2-phenylindole (DAPI), calcein acetomethoxyl (calcein-AM) and propidium iodide (PI) were purchased from KeyGen Biotech, Inc. (Nanjing). Cyanine5 amine dye (expressed as Cy5) was purchased from ApexBio Technology Company (Houston, USA). Roswell Park Memorial Institute (RPMI)-1640, highglucose dulbecco's modified eagle medium (DMEM), trypsin, phosphate buffer saline (PBS) and fetal bovine serum (FBS) were purchased from Gibco (Tulsa, OK). All chemicals were used as received without further purification. Human embryonic kidney cell line (HEK293 T cells, normal cells) and human lung adenocarcinoma cell line (A549 cells, tumor cells) were purchased from the Chinese Academy of Sciences Cell Bank (Shanghai, People's Republic of China). Animal care and handling procedures were in agreement with the guidelines evaluated and approved by the Animal Experiment Ethics Committee of Fudan University (2014-03-YJ-PZQ-01). 

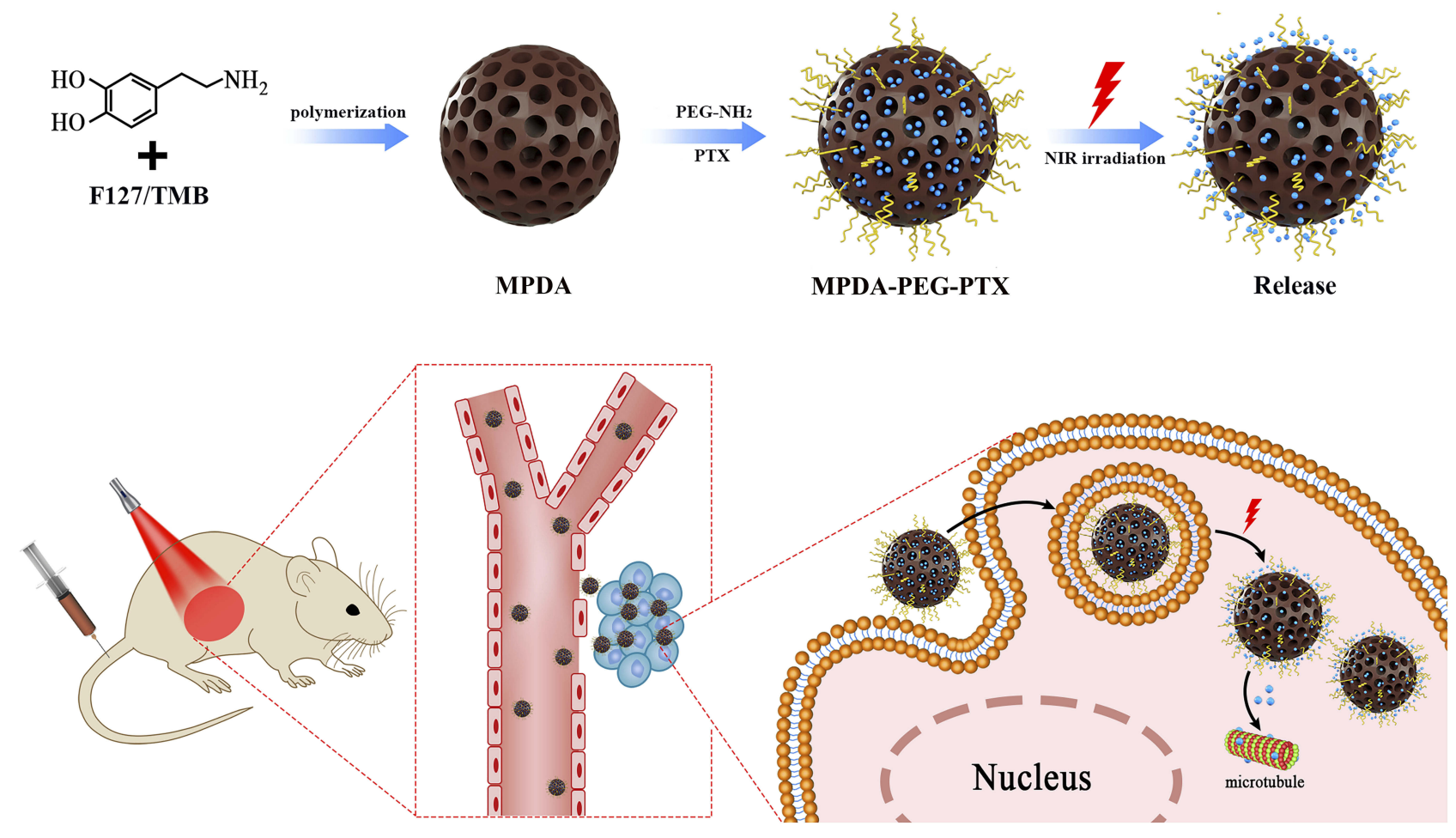

F127: PEO-PPO-PEO

MPDA: mesoporous polydopamine
TMB: 1, 3, 5 - Trimethylbenzene

PTX (paclitaxel): $\bullet \&$
PEG:

Scheme I Schematic illustration of the preparation of PEG-modified mesoporous polydopamine nanoparticles loaded with paclitaxel for chemo-photothermal synergistic therapy of tumor.

Abbreviation: PEG, polyethylene glycol.

\section{Characterization}

Transmission electron microscope (TEM) images were taken on a transmission electron microscope (Tecnai G2 20 TWIN, FEI, USA) operated at an acceleration of 10 $\mathrm{kV}$ by dropping solution onto a carbon-coated copper grid. Dynamic light scattering (DLS) and zeta potential were measured at $25^{\circ} \mathrm{C}$ on a Zetasizer Nano ZS90 analyzer (Malvern Instrument Ltd, UK). Brunauer-Emmett-Teller (BET) and Barrett-Joyner-Halenda (BJH) methods were used to test the surface area, the pore size distribution, and the pore volume. Fourier transform infrared (FT-IR) spectra were conducted on Thermofisher Nicolet 6700 fourier transform infrared spectrometer with $\mathrm{KBr}$ pellets. Raman spectra were performed on XploRA laser Raman spectrometer (HORIBA JobinYvon, France) with excitation of $532 \mathrm{~nm}$. Confocal laser scanning microscopy (CLSM) images were performed on Nikon $\mathrm{C} 2+$ laser scanning confocal microscope (Nikon, Japan). Photothermal effect was tested on an $808 \mathrm{~nm}$ consecutive NIR laser with spot size of
$5 \mathrm{~mm} \times 6 \mathrm{~mm}$ (Changchun New Industries Optoelectronics Technology Ltd., People's Republic of China). Thermal images were taken on an infrared camera thermographic system (Infra Tec, VarioCAM research)

\section{Synthesis Of Mesoporous Polydopamine (MPDA) Nanoparticles}

MPDA nanoparticles were synthesized using a modified reported method. ${ }^{28}$ In a typical reaction, $0.5 \mathrm{~g}$ of F127, $0.5 \mathrm{~g}$ of dopamine hydrochloride and $0.8 \mathrm{~mL}$ of TMB were dispersed in a mixture of $25 \mathrm{~mL}$ of water and $25 \mathrm{~mL}$ of ethanol by ultrasonication to forming an emulsion solution. Then, 2 $\mathrm{mL}$ of ammonia was added to the reaction mixture under stirring at $20^{\circ} \mathrm{C}$. The product was collected by centrifugation after 3-hr reaction and washed with water several times, then MPDA with size of ca. $190 \mathrm{~nm}$ was obtained. Various size of MPDA could be obtained by changing the reaction temperature $\left(40^{\circ} \mathrm{C}, 60^{\circ} \mathrm{C}\right.$ for $\sim 140,80 \mathrm{~nm}$, respectively). 


\section{Synthesis Of Nonporous Polydopamine (NPDA) Nanoparticles}

NPDA nanoparticles were synthesized using a modified reported method. ${ }^{29}$ In a typical synthesis of NPDA particles with size of $200 \mathrm{~nm}, 1 \mathrm{~mL}$ of ammonia was mixed with ethanol $(40 \mathrm{~mL})$ and deionized water $(90 \mathrm{~mL})$ under mild stirring at $30^{\circ} \mathrm{C}$ for $30 \mathrm{mins}$. Dopamine hydrochloride $(0.5 \mathrm{~g})$ was dissolved in deionized water $(10 \mathrm{~mL})$ and then injected into the above mixture solution. The reaction was allowed to proceed for $24 \mathrm{~h}$. NPDA particles were obtained by centrifugation and washed with water for several times, then NPDA nanoparticles with size of ca. $200 \mathrm{~nm}$ were obtained. The control over the size of NPDA was achieved by adjusting the amount of ammonia $(1.5,2 \mathrm{~mL}$ for $\sim 140,90 \mathrm{~nm}$, respectively).

MPDA/NPDA nanoparticles were modified by amineterminated PEG $\left(\mathrm{mPEG}-\mathrm{NH}_{2}, \mathrm{Mw}=4000\right)$. The obtained PEG-modified particles were purified by filtration through molecular weight 10,000 Da cut-off dialysis filters to remove excess $\mathrm{mPEG}-\mathrm{NH}_{2}$ and stored under $4{ }^{\circ} \mathrm{C}$ for further experiments. PEG-modified MPDA (MPDA-PEG) nanoparticles with diameter of $\sim 80,140$, and $190 \mathrm{~nm}$ are defined as MPDA-PEG-80, MPDA-PEG-140, MPDA-PEG-190, respectively.

\section{Measurement Of Photothermal Performance}

$100 \mu \mathrm{L}\left(100 \mu \mathrm{g} \mathrm{mL}^{-1}\right)$ of MPDA-PEG nanoparticles was dropped into a 96-well plate. Under illuminated $808 \mathrm{~nm}$ continuous-wave NIR laser $\left(2 \mathrm{~W} \mathrm{~cm}^{-2}\right)$ for $5 \mathrm{mins}$, the temperature was obtained by an infrared imaging camera. The photothermal conversion efficiency $(\eta)$ of MPDAPEG nanoparticles was determined according to the previous method. ${ }^{30}$ Detailed calculations were given as in the following:

$$
\begin{gathered}
\eta=\frac{h S \Delta T_{\max }-Q_{s}}{I\left(1-10^{-A}\right)}=\frac{h S\left(\Delta T_{\max }-\Delta T_{\operatorname{maxs}}\right)}{I\left(1-10^{-A}\right)} \\
h S=\frac{m_{s} C_{s}}{\tau}
\end{gathered}
$$

where $\mathrm{h}$ is the heat transfer coefficient, $\mathrm{S}$ is the surface area of the container, $\Delta \mathrm{T}_{\max }$ is the temperature change of the nanoparticle suspensions at the maximum steady-state temperature. $\Delta \mathrm{T}_{\max }$ represents the temperature change of solvent (e.g. $\mathrm{H}_{2} \mathrm{O}$ ) at the maximum steady-state temperature; $\mathrm{Q}_{s}$ is the heat associated with the NIR-light absorbance of the solvent. I is incident laser density $\left(2 \mathrm{~W} \mathrm{~cm}^{-2}\right)$, and $\mathrm{A}_{808}$ is the absorbance of the nanoparticles at $808 \mathrm{~nm}$. where $\tau$ is the sample system time constant, which can be determined by the linear curve fitting of temperature cooling time $v s$ its $\ln \frac{\Delta \mathrm{T}}{\Delta \mathrm{T}_{\max }}$. And $\mathrm{m}_{\mathrm{s}}$ and $\mathrm{C}_{\mathrm{s}}$ are the mass and the heat capacity of the solvent (pure water), respectively.

\section{Biodegradation Experiment}

Hydrogen peroxide solution $\left(\mathrm{H}_{2} \mathrm{O}_{2}, 5\right.$ or $\left.10 \mathrm{mM}\right)$ was added to MPDA nanoparticle suspension $\left(100 \mu \mathrm{g} \mathrm{mL}^{-1}\right)$ and stirred for reaction for $24 \mathrm{hrs}$. Afterwards, the absorbance at $808 \mathrm{~nm}$ was used for biodegradability evaluation.

\section{PTX Loading And Release}

Typically, $10 \mathrm{mg}$ of MPDA-PEG nanoparticles and $3 \mathrm{mg}$ of PTX were dispersed in $3 \mathrm{~mL}$ of acetone under stirring at room temperature. After $2 / 3$ volume of acetone was evaporated, PTX-loaded MPDA-PEG nanoparticles were then collected by centrifugation and washed with deionized water for three times to remove the surface adsorbed PTX. The PTX mass loaded in MPDA-PEG was calculated by UV-vis spectrophotometer at $228 \mathrm{~nm}$. The drugloading content (DLC) was calculated according to the following equations:

$$
\mathrm{DLC}(\%, \mathrm{PTX})=\frac{\text { weight of loaded PTX }}{\text { weight of PTX loaded nanoparticles }} \times 100 \%
$$

To investigate the PTX release performance, $5 \mathrm{mg}$ of PTXloaded MPDA-PEG-190 (MPDA-PEG-PTX) nanoparticles were dispersed in $5 \mathrm{~mL}$ of PBS. Each sample was then transferred into a dialysis bag, which was dialyzed against $20 \mathrm{~mL}$ of the corresponding buffer and shaking under dark. At predetermined periods, $2 \mathrm{~mL}$ of solution was withdrawn from the solution, and $2 \mathrm{~mL}$ of fresh PBS was added. All drug release results were averaged with three repeated measurements. To investigate the effect of NIR irradiation on the drug release performance, MPDA-PEG-PTX $\left(1 \mathrm{mg} \mathrm{mL}^{-1}\right)$ was incubated under NIR irradiation, concentration of released PTX was measured by UV-vis absorbance.

\section{In Vitro Cytotoxicity Assay}

HEK-293T cells (human embryonic kidney cell line) were cultured in DMEM containing 10\% (v/v) FBS. 293T cells were plated in 96-well culture plates for 24-hr incubation. Subsequently, the cells are treated with different concentrations of MPDA-PEG nanoparticle dispersion and incubated for $24 \mathrm{hrs}$. Finally, the standard 3-(4,5-dimethylthiazol-2yl)-2,5-diphenyltetrazolium bromide (MTT) assay was used to measure the viability of the cells. 


\section{In Vitro PTT And Chemotherapeutic Assay}

A549 cells (human lung adenocarcinoma cell line) cultured with RPMI-1640 culture medium (containing 10\% (v/v) FBS) were seeded in 96-well plates for 24-hr incubation. For MPDA-PEG + Laser and MPDA-PEG-PTX + Laser group, A549 cells were treated with MPDA-PEG/MPDAPEG-PTX nanoparticles for $4 \mathrm{~h}$ and irradiated by NIR laser $\left(808 \mathrm{~nm}, 2 \mathrm{~W} \mathrm{~cm}^{-2}\right)$ for 5 mins. And then the therapeutic effect is measured after another 24-hr incubation. For measuring the therapeutic efficacy of MPDA-PEG, PTX and MPDA-PEG-PTX group, MPDA-PEG, free PTX and MPDA-PEG-PTX nanoparticle were added to A549 cells and incubate $24 \mathrm{~h}$ directly.

\section{Cellular Uptake Assay}

For cellular uptake assay, Cy5 modified MPDA-PEG nanoparticles were prepared by mixing $20 \mathrm{mg}$ of MPDA-PEG nanoparticles with $5 \mathrm{mg}$ of amine-terminated $\mathrm{Cy} 5$ in dimethyl sulfoxide (DMSO)/PBS (v/v, 1/99) solution by magnetic stirring for $24 \mathrm{hrs}$ in dark. The actual loading amount of Cy5 on the surface of MPDA-PEG particles was $1 / 9$ (weight/weight), quantified by measuring the fluorescence intensity of free Cy5 $\left(\lambda_{\mathrm{ex}}=640 \mathrm{~nm}, \lambda_{\mathrm{em}}=665 \mathrm{~nm}\right)$ in the supernatant. The cellular uptake of Cy5 modified MPDA-PEG-PTX nanoparticles was measured by confocal laser scanning microscopy (CLSM). A549 cells were cultured in 35-mm glass bottomed dishes for $12 \mathrm{hrs}$, Cy5 modified MPDA-PEG-PTX nanoparticles was added into dishes co-incubated for another $4 \mathrm{hr}$. Afterwards, cells were stained with 4, 6-diamidino-2-phenylindole (DAPI, nuclear staining reagent) for 15 mins. Fluorescence images of cells were captured by CLSM, the $\lambda_{\mathrm{ex}} / \lambda_{\mathrm{em}}$ of DAPI is $359 / 461 \mathrm{~nm}$.

\section{Live/dead Cell Viability Assay}

A549 cells were cultured in 35-mm glass bottomed dishes for $12 \mathrm{hrs,} \mathrm{PTX,} \mathrm{MPDA-PEG} \mathrm{and} \mathrm{MPDA-PEG-PTX} \mathrm{sus-}$ pension was added into dishes co-incubated for another 4 hrs, then A549 cells were irradiated under $808 \mathrm{~nm}$ laser for 5 mins. Afterwards, cells were stained with calcein acetomethoxyl (calcein-AM) and propidium iodide (PI) for 15 mins. Fluorescence images of cells were captured by CLSM, the $\lambda_{\text {ex }} / \lambda_{\text {em }}$ of calcein-AM and PI are 499/515 $\mathrm{nm}$ and 495/635 $\mathrm{nm}$, respectively.

\section{In Vitro And In Vivo PAI}

For in vitro PAI, different concentrations of MPDA-PEG nanoparticles were filled in the polyethylene tube and then measured by the high-resolution preclinical PAI system. For in vivo PAI, tumor-bearing mice were intravenously injected with $100 \mu \mathrm{L}$ ( $4 \mathrm{mg} \mathrm{mL}^{-1}$ ) of MPDA-PEG-PTX. Subsequently, the PA signal of the tumor at different time points post-injection was captured by the high-resolution preclinical PAI system.

\section{Pharmacokinetics And Biodistribution Study} Four Balb/c nude mice (male, 20-22 g) were treated with $100 \mu \mathrm{L}$ of Cy5 modified MPDA-PEG-PTX nanoparticles (4 $\mathrm{mg} \mathrm{mL}^{-1}$ ) intravenously. At different time points postinjection, $100 \mu \mathrm{L}$ of blood were collected from mouse orbit and diluted to $1 \mathrm{~mL}$. The blood samples were centrifuged at $1200 \mathrm{rpm}$, and the supernatant was collected for fluorescence intensity measurement. Subsequently, eight A549 tumor-bearing mice were treated with $100 \mu \mathrm{L}$ of Cy5 modified MPDA-PEG-PTX (4 mg mL $\mathrm{mL}^{-1}$ ) intravenously. At 4-hr and 24-hr post-injection, each four mice were sacrificed, their tumors and major organs were harvested and homogenized in lysis buffer. Afterwards, the lysate of each sample was centrifuged and the supernatant was subjected to fluorescence intensity measurement.

\section{In Vivo Therapy}

When the tumor volume reached to $80 \mathrm{~mm}^{3}$, mice were separated into six groups $(\mathrm{n}=5)$ : Control group (Group 1), PTX group (Group 2), MPDA-PEG-PTX group (Group 3), MPDA-PEG $+2 \mathrm{~W} \mathrm{~cm}^{-2}$ laser group (5 mins, Group 4), MPDA-PEG-PTX $+2 \mathrm{~W} \mathrm{~cm}^{-2}$ Laser group ( 5 mins, Group 5 ) and MPDA-PEG-PTX $+1 \mathrm{~W} \mathrm{~cm}^{-2}$ Laser group (10 mins, Group 6). For group 2, mice were injected intravenously with $100 \mu \mathrm{L}$ free PTX $\left(0.6 \mathrm{mg} \mathrm{mL}^{-1}\right)$. For groups $3-6$, mice were injected intravenously with $100 \mu \mathrm{L}$ MPDA-PEG/MPDAPEG-PTX nanoparticle suspension $\left(4 \mathrm{mg} \mathrm{mL}^{-1}\right)$. At 4-hr post-injection, the tumors were exposed to $808 \mathrm{~nm}$ NIR laser. The temperature of tumor site was recorded by an infrared camera. The size of tumors and mice weights were measured every other day. At 14th day, all of the mice were euthanized and the tumors and major organs (heart, liver, spleen, lung, and kidney) were dissected and stained with hematoxylin and eosin (H\&E) for histological analysis.

\section{Blood Analysis}

To test the in vivo biosafety, ICR mice (male, 20-22 g) were divided into three groups: Control group, MPDA-PEG group and MPDA-PEG-PTX group. MPDA-PEG group and MPDA-PEG-PTX group were treated with $100 \mu \mathrm{L}$ of MPDA-PEG/MPDA-PEG-PTX (4 $\left.\mathrm{mg} \mathrm{mL}^{-1}\right)$ intravenously. 
At 48-hr post-injection, mice were sacrificed and their blood samples were collected. The whole blood panel data were tested. Three important hepatic indicators and two renal function-related indicators were measured using a 7080 blood biochemical auto analyzer (Hitachi, Japan).

\section{Statistical Analysis}

All data in this paper are expressed as mean result \pm SD. Unpaired student's $t$-test was used for comparison between two testing groups and a probability $(P)$ less than 0.05 was considered statistical significance.

\section{Results And Discussion Preparation And Characterization Of MPDA-PEG}

MPDA with large mesochannel is prepared by emulsioninduced interface assembly strategy with slightly modified. ${ }^{28}$
Transmission electron microscopy (TEM) images of three diameters of MPDA ( 80, 140, $190 \mathrm{~nm})$ nanoparticles are shown in Figure 1A, MPDA nanoparticles exhibit uniform particle size distribution and the mesochannel arranged radially. TEM image with a higher magnification (Figure S1) reveals that the pore size estimated to be $\sim 15 \mathrm{~nm}$. The hydrodynamic size of MPDA (Table 1) measured from dynamic light scattering (DLS) is a little bigger than observed under TEM, which is caused by hydration layer on the surface of nanoparticles. ${ }^{31}$ To enhance their colloidal stability in physiological medium for further biological applications, amine-terminated PEG has introduced to functionalize MPDA under $\mathrm{pH}$ 12.0 via the Michael addition and/or Schiff base reaction. ${ }^{32}$ The $\zeta$-potential of PEG-modified MPDA (MPDA-PEG) nanoparticles changes from $-37 \mathrm{mV}$ to $-9.4 \mathrm{mV}$ proves that $\mathrm{PEG}$ has been successfully modified to MPDA nanoparticles, which may contribute to prolonged drug circulation time. ${ }^{33}$ DLS

a)
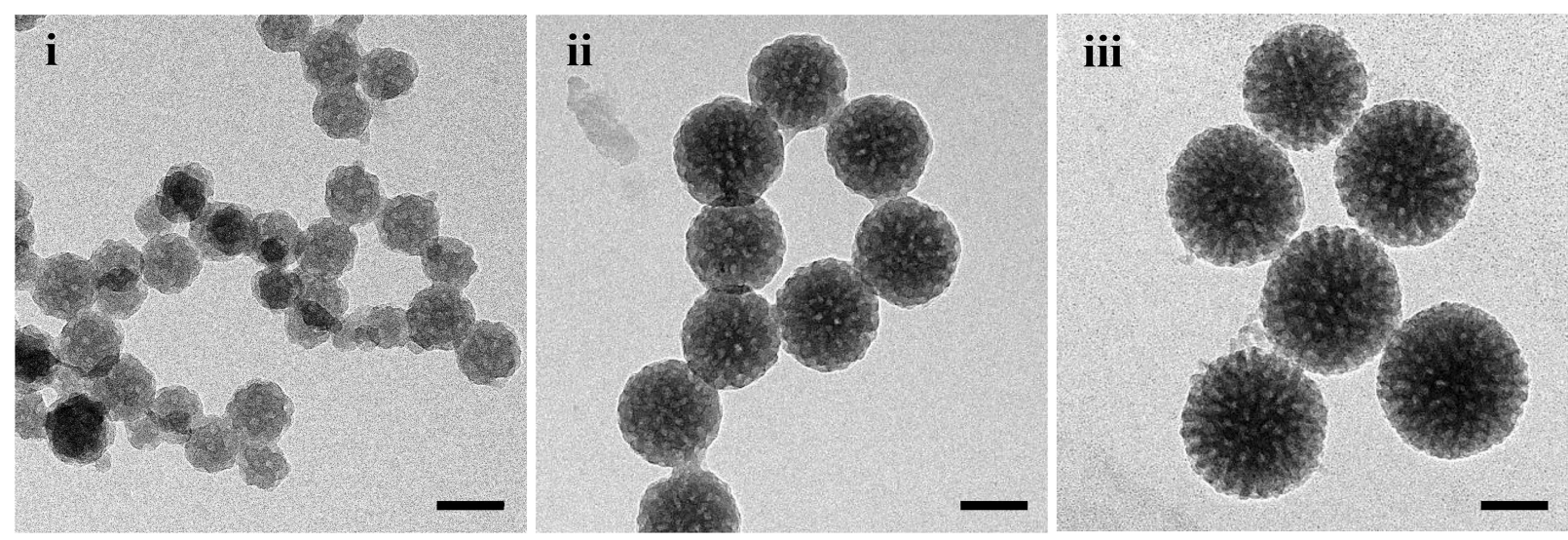

b)

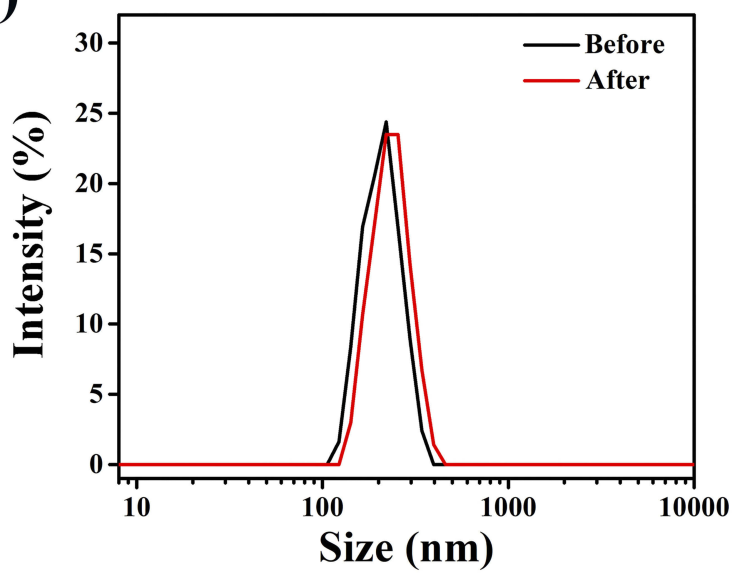

c)
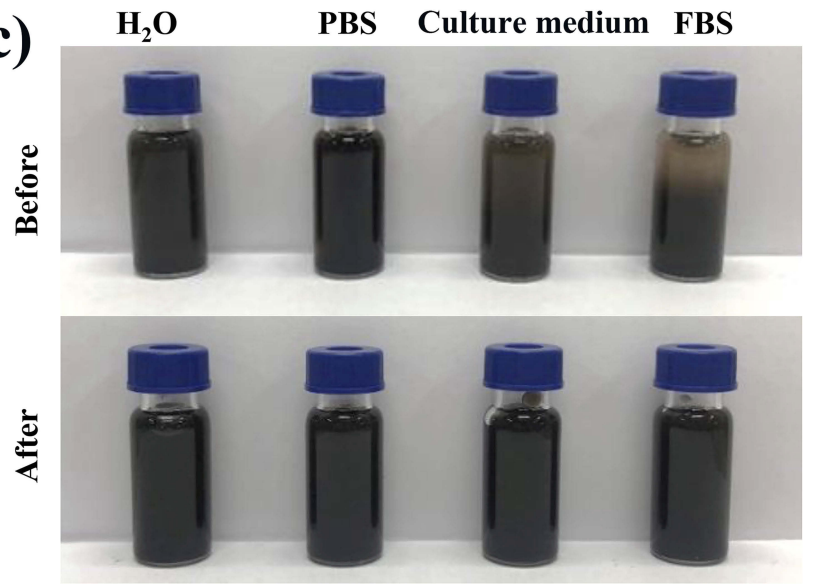

Figure I (a) TEM images of MPDA nanoparticles: (i) $\sim 80 \mathrm{~nm}$, (ii) $\sim 140 \mathrm{~nm}$, and (iii) $\sim 190 \mathrm{~nm}$. The scale bars are $100 \mathrm{~nm}$. (b) Hydrodynamic diameters of MPDA ( 190 nm) before and after PEG modified. (c) Photographs of MPDA before and after PEG modified in different media (water, PBS, RPMI-I640 medium (containing I0\% FBS), FBS) for 4 hrs.

Abbreviations: TEM, transmission electron microscope; MPDA, mesoporous polydopamine; PEG, polyethylene glycol; PBS, phosphate buffer saline; RPMI, Roswell Park Memorial Institute; FBS, fetal calf serum. 
Table I The Colloidal Data Of MPDA Nanoparticles Before And After PEG Modified

\begin{tabular}{|l|l|l|l|l|}
\hline Sample & $\begin{array}{l}\mathbf{D}_{\text {TEM }} \\
(\mathbf{n m})^{\mathbf{a}}\end{array}$ & $\begin{array}{l}\mathbf{D}_{\mathbf{h}} \\
\mathbf{( n m})^{\mathbf{b}}\end{array}$ & PDI $^{\mathbf{c}}$ & $\begin{array}{l}\text { Zeta } \\
\text { Potential } \\
(\mathbf{m V})\end{array}$ \\
\hline MPDA-80 & $83 \pm 5$ & 113 & 0.042 & -39.2 \\
MPDA-140 & $141 \pm 7$ & 170 & 0.043 & -35.9 \\
MPDA-190 & $194 \pm 10$ & 219 & 0.061 & -37.4 \\
MPDA-PEG-80 & - & 127 & 0.050 & -8.6 \\
MPDA-PEG-140 & - & 184 & 0.077 & -6.8 \\
MPDA-PEG-190 & - & 232 & 0.038 & -9.4 \\
\hline
\end{tabular}

Notes: ${ }^{\text {a }} \mathrm{D}_{\text {TEM, }}$ average diameter by measuring 30 nanoparticles from TEM images. ${ }^{\mathrm{b}}$ The hydrodynamic diameter measured with dynamic light scattering in water. 'Polydispersity index of the particle size, PDI $=\left\langle\mu_{2}\right\rangle \mid \Gamma^{2}$.

Abbreviations: TEM, transmission electron microscope; PDI, polydispersity index MPDA, mesoporous polydopamine; MPDA-PEG, polyethylene glycol-modified mesoporous polydopamine.

a)

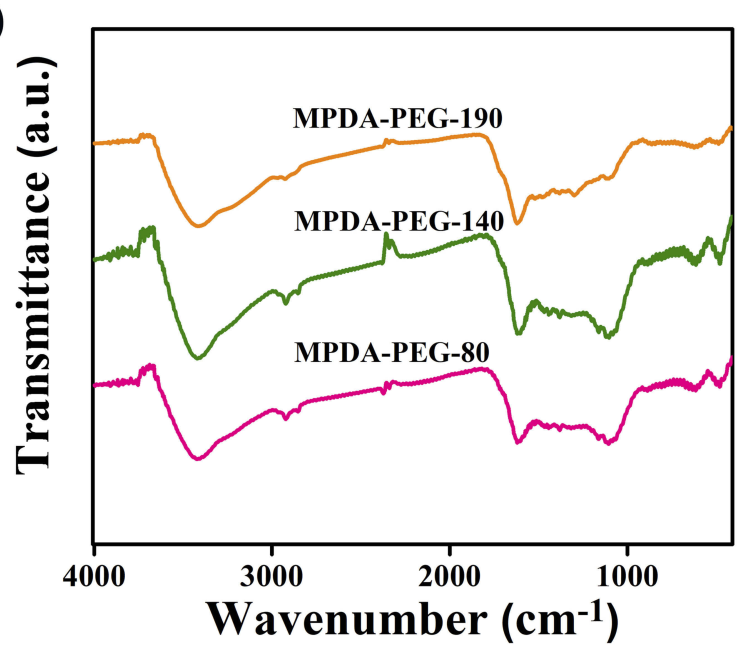

c)

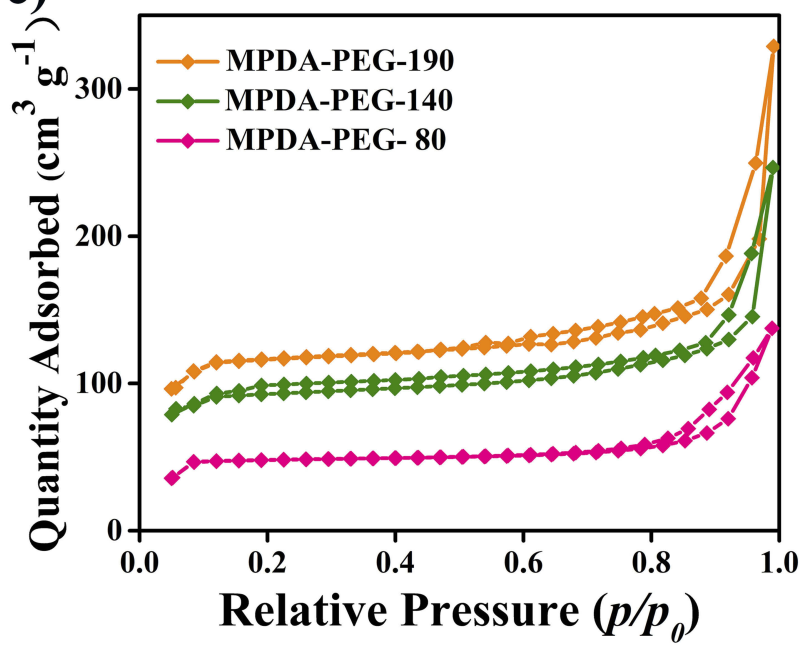

results also illustrate the increase of hydrodynamic size of MPDA nanoparticles after PEG-modified (Figure 1B and Table 1). As shown in Figure 1C, MPDA nanoparticles after PEG modified exhibit better stability in physiological medium (RPMI-1640 medium (containing 10\% FBS) and FBS) for 4 hrs, which is conducive to further biological applications. MPDA-PEG nanoparticles with various diameters (about 80, 140 and $190 \mathrm{~nm}$ ) are defined as MPDA-PEG-80, MPDAPEG-140 and MPDA-PEG-190, respectively.

MPDA-PEG nanoparticles with various diameters are then characterized by Fourier transform infrared spectra (FT-IR), Raman spectra, and nitrogen adsorption-desorption isotherms. The FT-IR spectra are shown in Figure $2 \mathrm{~A}$, the broad band around $3400 \mathrm{~cm}^{-1}$ is ascribed

b)

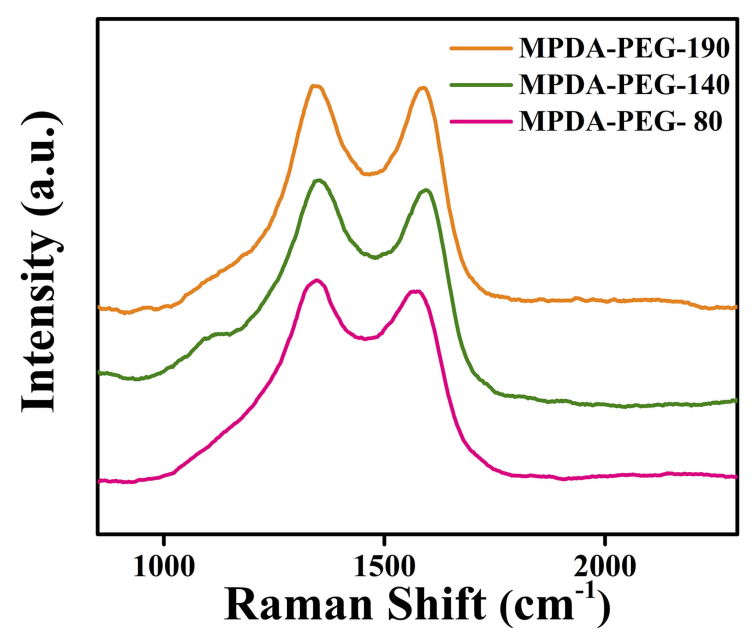

d)

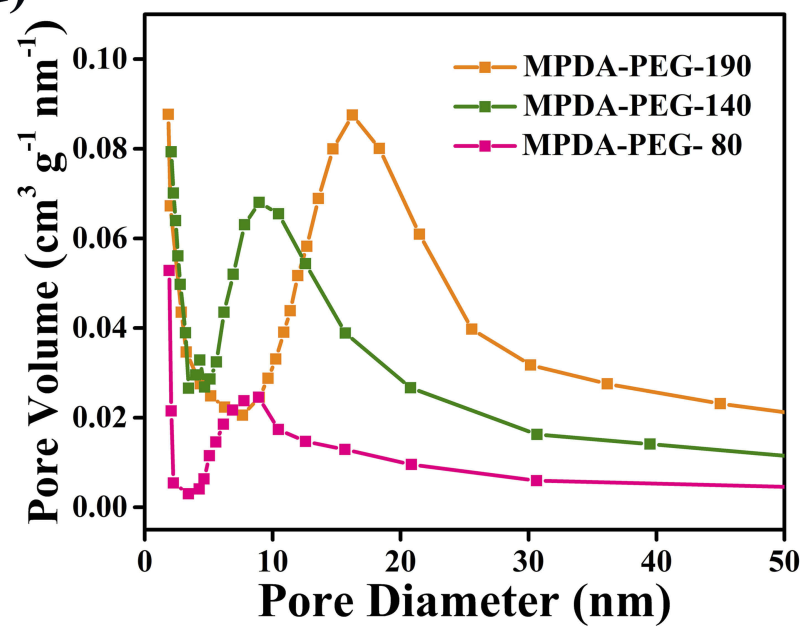

Figure 2 (a) FT-IR spectra and (b) Raman spectrum of MPDA-PEG nanoparticles. (c) Nitrogen adsorption-desorption isotherms and (d) pore size distribution curves of MPDA-PEG nanoparticles with various diameters.

Abbreviation: MPDA-PEG, polyethylene glycol-modified mesoporous polydopamine. 
to the stretching vibration of $\mathrm{N}-\mathrm{H}$ and $\mathrm{O}-\mathrm{H}$ hydroxyl, the clear peak at $1616 \mathrm{~cm}^{-1}$ is the stretching vibration of $\mathrm{C}=\mathrm{C}$ double bonds from aromatic ring. ${ }^{34}$ These functional groups endow MPDA-PEG nanoparticles with an active surface for modification. As shown in Figure 2B, Raman spectroscopy is used to provide information on the disordered and defect structure of MPDA-PEG nanoparticles. ${ }^{35}$ Two strong peaks centered at 1350 and $1590 \mathrm{~cm}^{-1}$ are observed, the vibration signal at $1350 \mathrm{~cm}^{-1}$ is attributed to $\mathrm{sp} 2$ carbon of 2D hexagonal structure lattice (D band) while the vibration signal at $1590 \mathrm{~cm}^{-1}$ corresponded to $\mathrm{sp} 2$ carbon of in-plane aromatic structure ring ( $\mathrm{G}$ band). ${ }^{36}$ The Raman intensity of $\mathrm{D}$ band versus that of $\mathrm{G}$ band is very close to 1 , indicating that the obtained mesoporous polydopamine nanoparticles may have a graphene sheetlike structure with high-density vacancy defects. ${ }^{37}$ Pore volume of mesoporous material is one of the most significant factors affecting DLC. Nitrogen adsorption-desorption isotherms experiment is used to testify the regularity of pore characteristics with the growth of particle size. As shown in Figure $2 \mathrm{C}$ and $\mathrm{D}$, the specific surface area of MPDA-PEG-80 is $68 \mathrm{~m}^{2} \mathrm{~g}^{-1}$, cumulative pore volume is $0.16 \mathrm{~cm}^{3} \mathrm{~g}^{-1}$. With the particle size increasing from 80 to $190 \mathrm{~nm}$, the specific surface area is gradually expanded to $286 \mathrm{~m}^{2} \mathrm{~g}^{-1}$, while cumulative pore volume is $0.32 \mathrm{~cm}^{3} \mathrm{~g}^{-1}$, which is beneficial for loading of chemotherapeutic drugs. Nitrogen adsorption-desorption isotherms experiments prove the enhanced specific surface area and pore volume of MPDA-PEG with the increase of particle diameter, which is probably caused by the gradual improvement and perfection of open framework structure with the size growth. ${ }^{38}$

\section{Photothermal Effect Of MPDA-PEG}

Nanomaterials with high NIR absorption and photothermal conversion efficiency for PTT are highly desirable. ${ }^{39}$ To investigate the photothermal effect of MPDA-PEG nanoparticles, PEG-modified NPDA (NPDA-PEG) nanoparticles (diameter of about 90, 140, $200 \mathrm{~nm}$, defined as NPDA-PEG-90, NPDAPEG-140 and NPDA-PEG-200) are synthetized for comparison (TEM, FT-IR, Raman and UV-vis spectra are packed in

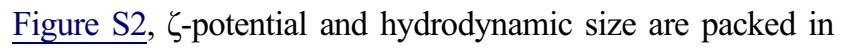
Table S1). Then, MPDA-PEG and NPDA-PEG nanoparticles $\left(100 \mu \mathrm{g} \mathrm{mL}^{-1}\right)$ are exposed to NIR laser $\left(808 \mathrm{~nm}, 2 \mathrm{~W} \mathrm{~cm}^{-2}\right)$ for $5 \mathrm{~min}$, the increases of temperature are recorded by an infrared thermal camera. As shown in Figure 3A, MPDAPEG-190 nanoparticles can elevate the temperature from $25^{\circ}$ $\mathrm{C}$ to $66.5^{\circ} \mathrm{C}\left(\Delta \mathrm{T}=41.5^{\circ} \mathrm{C}\right)$, which is obviously higher than
MPDA-PEG-140 $\left(\Delta \mathrm{T}=34.8^{\circ} \mathrm{C}\right)$ and MPDA-PEG-80 $(\Delta \mathrm{T}=$ $27.8^{\circ} \mathrm{C}$ ). Therefore, MPDA-PEG nanoparticles own an enhanced photothermal effect with size increase, which is consistent with our previous research on natural melanin nanoparticles and metal-organic framework (MOF)-derived carbon nanospheres. ${ }^{24,40}$ Meanwhile, NPDA-PEG nanoparticles exhibit lower temperature increment than MPDA-PEG nanoparticles (Figure S3). The temperature of NPDAPEG-200 nanoparticles suspension is elevated to $58.5^{\circ} \mathrm{C}$ $\left(\Delta \mathrm{T}=33.5^{\circ} \mathrm{C}\right)$ at concentration of $100 \mu \mathrm{g} \mathrm{mL}{ }^{-1}$, which is obvious lower than MPDA-PEG-190, suggesting that MPDAPEG nanoparticles have better photothermal performance than NPDA-PEG.

To reveal the excellent photothermal effect of MPDAPEG-190, two factors are considered: the light absorbance in NIR region and photothermal conversion efficiency $(\eta){ }^{41}$ As shown in Figure $3 \mathrm{~B}$ and $\mathrm{C}$, UV-vis spectra indicate that the NIR absorbance of MPDA-PEG at 808 $\mathrm{nm}$ increases gradually with the growth of the particle size. Meanwhile, the $\eta$ values of MPDA-PEG-80, MPDA-PEG140 and MPDA-PEG-190 are calculated as 37.6\%, 40.1\% and $41.2 \%$, respectively (Figure $3 \mathrm{D}$ ), ${ }^{30}$ while the $\eta$ values of NPDA-PEG nanoparticles (NPDA-PEG-90 (26.8\%), NPDA-PEG-140 (30.3\%), NPDA-PEG-200 (33.1\%)) are obviously lower than MPDA-PEG. More importantly, the $\eta$ value of MPDA-PEG is higher than most other photothermal agents (Table S2). ${ }^{42}$ Therefore, owing to the enhanced NIR absorbance and photothermal conversion efficiency with the increase of particles, MPDA-PEG-190 displays the best photothermal effect, which could be used for future research.

In addition, the photothermal stability of MPDA-PEG nanoparticles is examined by repeated exposure to NIR laser irradiation $\left(808 \mathrm{~nm}, 2 \mathrm{~W} \mathrm{~cm}^{-2}\right.$ ) for five cycles. Little loss of thermal fatigue resistance observed over five cycles of exposure to NIR laser (Figure S4). Meanwhile, negligible changes in absorption spectra are observed after five cycles of irradiation, demonstrating the good photothermal stability of MPDA-PEG nanoparticles. As possessing the best photothermal effect, MPDA-PEG-190 nanoparticles are used in subsequent experiments.

Subsequently, the biodegradability of MPDA-PEG-190 nanoparticles is evaluated in the presence of hydrogen peroxide $\left(\mathrm{H}_{2} \mathrm{O}_{2}\right)$, which is widely distributed in macrophages and major organs. ${ }^{43}$ The absorption of MPDAPEG-190 at $808 \mathrm{~nm}$ declined along with reaction time and increasing concentration of $\mathrm{H}_{2} \mathrm{O}_{2}$. The degradation $\mathrm{t}_{1 / 2}$ value of MPDA-PEG-190 reacted with $10 \mathrm{mM} \mathrm{H}_{2} \mathrm{O}_{2}$ 

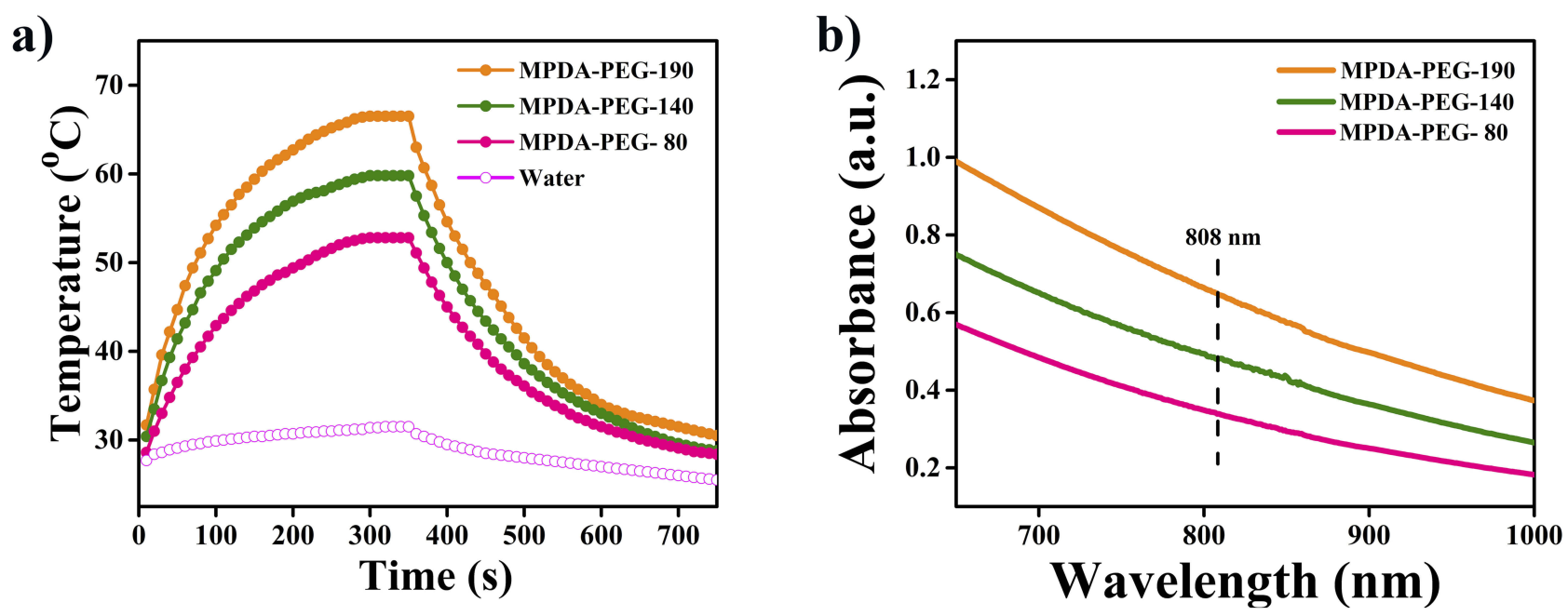

c)
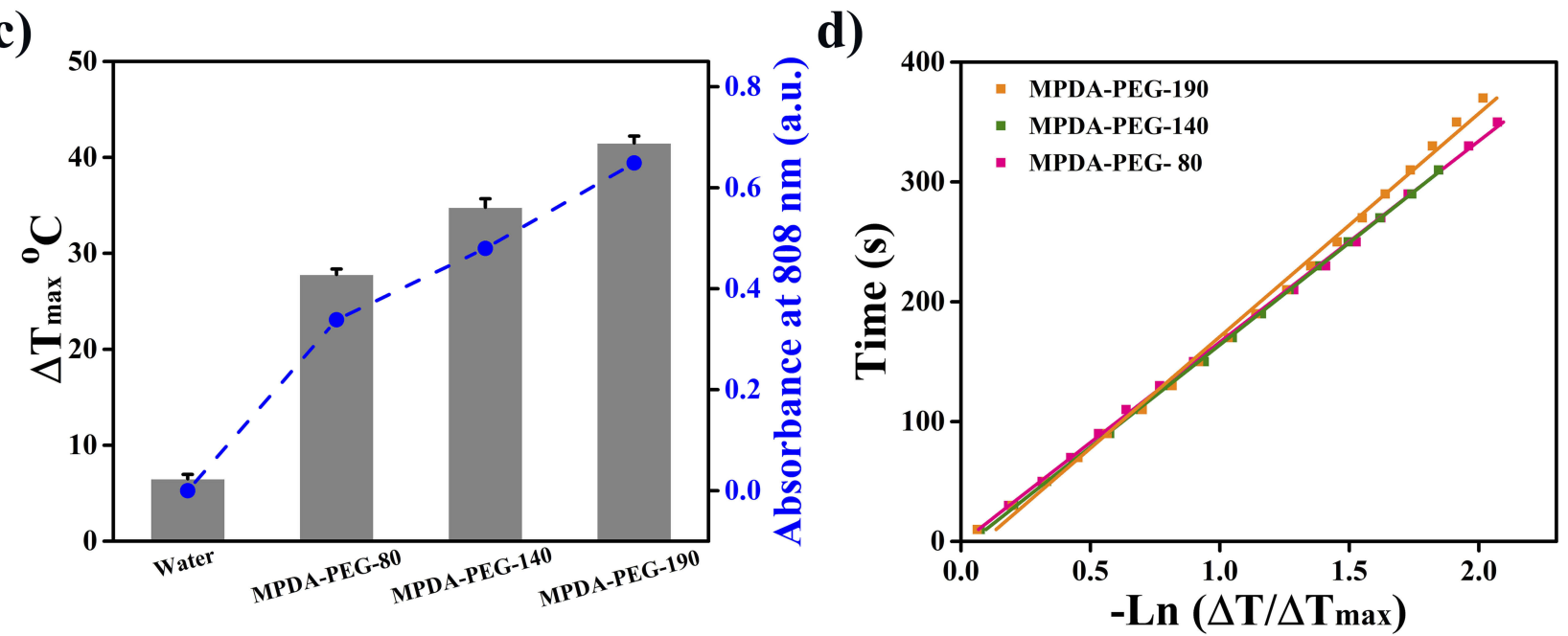

Figure 3 (a) Photothermal effects of MPDA-PEG nanoparticle under laser irradiation ( $100 \mu \mathrm{g} \mathrm{mL} \mathrm{L}^{-1}, 808 \mathrm{~nm}, 2 \mathrm{~W} \mathrm{~cm}^{-2}$ ) for $5 \mathrm{mins}$. (b) UV-vis absorbance spectra of MPDAPEG nanoparticles at a concentration of $100 \mu \mathrm{gL}^{-1}$. (c) Summary of the maximum temperature change of MPDA-PEG during NIR laser exposure, together with their absorbance at $808 \mathrm{~nm}$. (d) Linear time data versus - $\mathrm{Ln}\left(\Delta \mathrm{T} / \Delta \mathrm{T}_{\max }\right)$ obtained from the cooling periods of Figure $3 \mathrm{~A}$.

Abbreviations: MPDA-PEG, polyethylene glycol-modified mesoporous polydopamine; NIR, near Infrared.

is calculated to be $5.9 \mathrm{hrs}$ (Figure S5), which is faster than NPDA-PEG-200 $\left(t_{1 / 2}, 7.2 \mathrm{~h}\right)$. These results indicated that MPDA-PEG nanoparticles have good photothermal effect and biodegradability.

\section{Drug Loading And Release}

With expected mesoporous structure, MPDA-PEG could be regarded as admirable drug delivery vehicles for PTX. ${ }^{44}$ Then, PTX loading ability of MPDA-PEG-190 nanoparticles is been further investigated. As shown in Figure 4A, successful loading of PTX is confirmed by FT-IR spectra. Spectrum of PTX shows characteristic absorptions at 1718 and $1244 \mathrm{~cm}^{-1}$, which is ascribed to the symmetric or asymmetric stretch of $\mathrm{C}=\mathrm{O}$ and $\mathrm{C}-\mathrm{O}$, respectively. ${ }^{45}$ As for MPDA-PEG particles after loading with PTX, the new peak located at
$1718 \mathrm{~cm}^{-1}$ along with $1244 \mathrm{~cm}^{-1}$ corroborate that PTX has been loaded into the MPDA-PEG. Nitrogen adsorption-desorption isotherms experiments of MPDA-PEG-190 and PTX-loaded MPDA-PEG-190 (MPDA-PEG-PTX) nanoparticles (Figure 4B and $\mathrm{C}$ ) suggest the surface area and pore volume are decreased from $286 \mathrm{~m}^{2} \mathrm{~g}^{-1}$ and $0.32 \mathrm{~cm}^{3} \mathrm{~g}^{-1}$ to $44 \mathrm{~m}^{2} \mathrm{~g}^{-1}$ and $0.12 \mathrm{~cm}^{3} \mathrm{~g}^{-1}$ respectively, which dues to the successful loading of PTX into the mesoporous channels of MPDA-PEG nanoparticles. Subsequently, the DLC of MPDA-PEG nanoparticles and NPDA-PEG nanoparticles are further compared. It found that only a small portion of PTX molecules are adsorbed onto the surface of NPDAPEG-200 nanoparticles (DLC, 3.8\%), while MPDA-PEG190 could load PTX molecules effectively (DLC, 15\%), drug-loading capacity increased nearly four times. Hence, 
a)

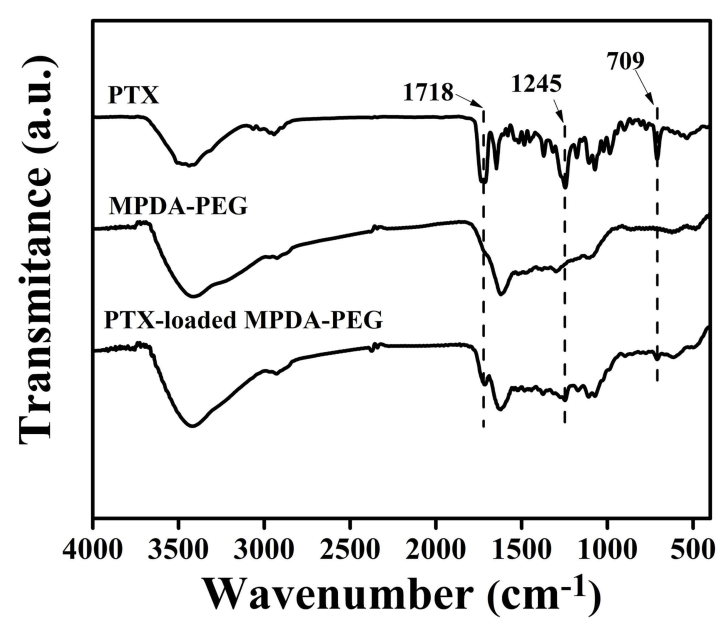

c)

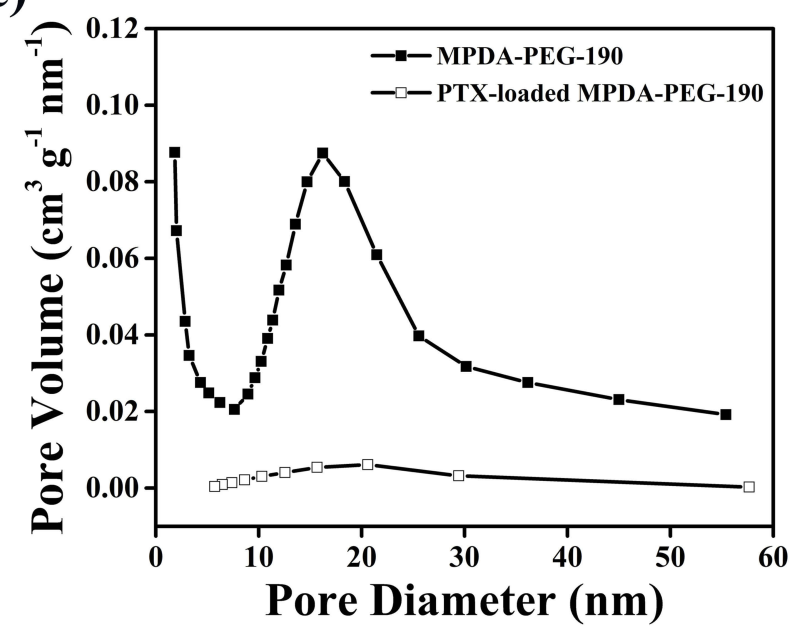

b)

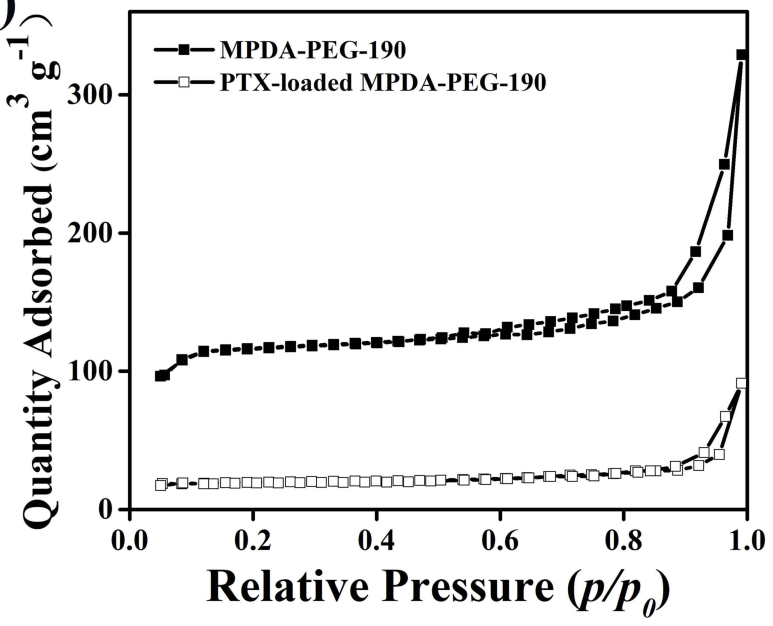

d)

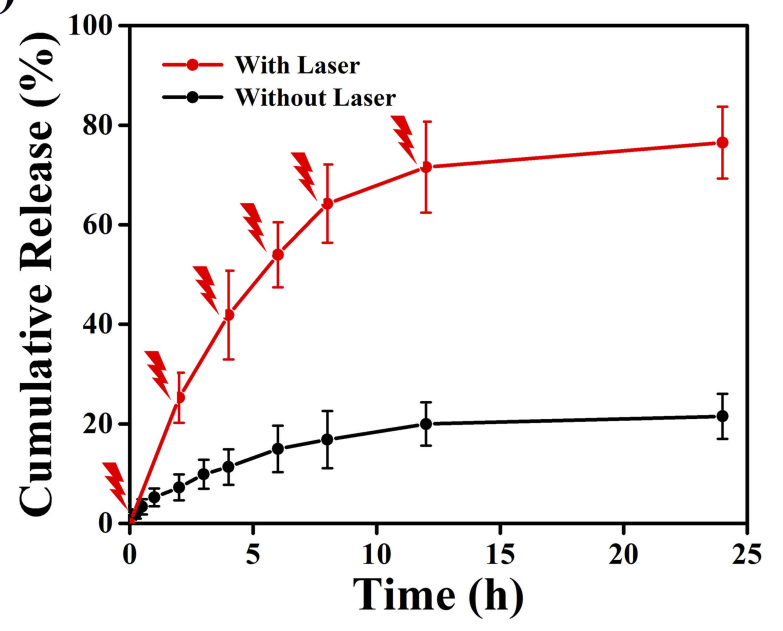

Figure 4 (a) FT-IR spectra of PTX, MPDA-PEG and PTX-loaded MPDA-PEG nanoparticles. (b) Nitrogen adsorption-desorption isotherms and (c) pore size distribution curves of MPDA-PEG-190 and PTX-loaded MPDA-PEG-190. (d) PTX release performance of PTX-loaded MPDA-PEG-I90 with or without NIR irradiation (808nm, 2 W $\mathrm{cm}^{-2}, 5$ mins) at an interval of $2 \mathrm{hrs}$.

Abbreviations: PTX, paclitaxel; MPDA-PEG, polyethylene glycol-modified mesoporous polydopamine; NIR, near Infrared.

the drug-loading efficiency of PTX could be improved significantly based on mesoporous structure.

The PTX release behavior of MPDA-PEG-PTX nanoparticles is further evaluated. Figure 4 D shows that about $20 \%$ of PTX released from MPDA-PEG-PTX nanoparticles during 24 hrs. This result indicates a high affinity and subsequent tight retention of the surface of MPDA-PEG nanoparticles toward PTX molecules, which is beneficial for preventing premature release and result in sustained in vivo therapeutic effect. ${ }^{46}$ To further understand the influence of NIR irradiation on PTX release performance, MPDA-PEG-PTX nanoparticle dispersion is irradiated with $808 \mathrm{~nm}$ laser $\left(2 \mathrm{~W} \mathrm{~cm}^{-2}, 5\right.$ mins $)$ at an interval of 2 hrs. As shown in Figure 4D, nearly $80 \%$ of PTX molecules are released from MPDA-PEG-PTX nanoparticles eventually. The drastic release of PTX is mainly due to the rapid temperature increasing of MPDA-PEG dispersion under NIR irradiation, which facilitates the PTX molecule thermal motion and the escape of PTX molecules from the mesochannels of MPDA-PEG nanoparticles. ${ }^{12}$ The above result clearly indicates that MPDA-PEG-PTX nanoparticles exhibit NIRresponsive drug release behavior, the elevated temperature can facilitate the release of chemotherapeutic drugs in tumor site, increasing the bioavailability of PTX and decreasing the side effect, producing synergistic therapeutic effect. ${ }^{47-50}$

\section{In Vitro Chemo-Photothermal Therapy}

Encouraged by the excellent photothermal effect and exceptional PTX loading capacity of MPDA-PEG nanoparticles, its bio-application is further investigated. Firstly, the biocompatibility of MPDA-PEG $\left(5-200 \mu \mathrm{g} \mathrm{mL}^{-1}\right)$ is measured 
after incubation with HEK-293T cells for $24 \mathrm{hrs}$. As shown in Figure $5 \mathrm{~A}$, the cell viabilities of all groups are over $90 \%$, even at high concentration $\left(200 \mu \mathrm{g} \mathrm{mL}^{-1}\right)$, indicating the excellent biocompatibility of blank MPDA-PEG. To further confirm the synergistic therapeutic effect of MPDA-PEGPTX nanoparticles, A549 cells are incubated with MPDAPEG, free PTX and MPDA-PEG-PTX at various concentrations. As shown in Figure 5B, the cell viabilities of A549 cells incubated with various concentration MPDAPEG are all over 95\%, indicating blank MPDA-PEG does little harm to cancer cells. The cytotoxicity of MPDA-PEGPTX group is significantly lower than free PTX group at equivalent concentration of PTX, which should be attributed to the sustained release of PTX molecules. Although

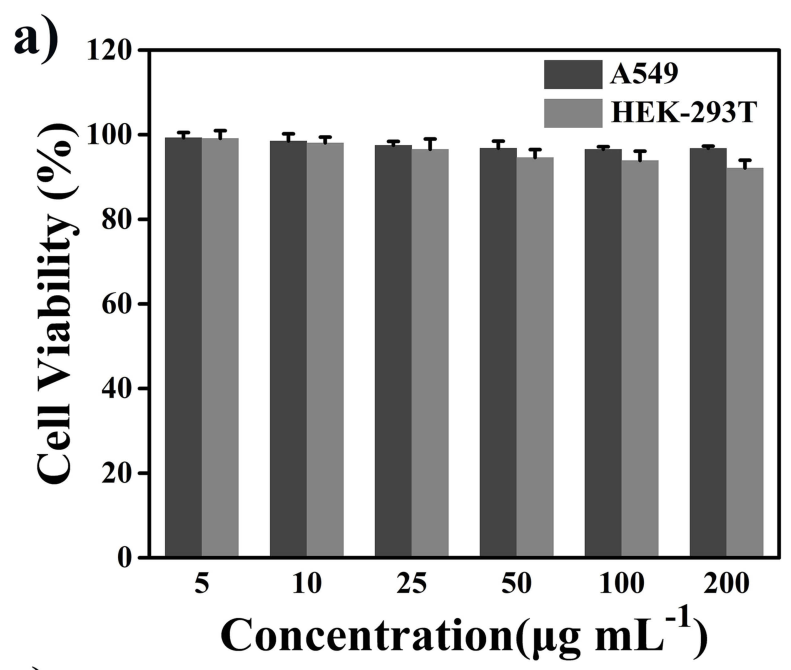

c)
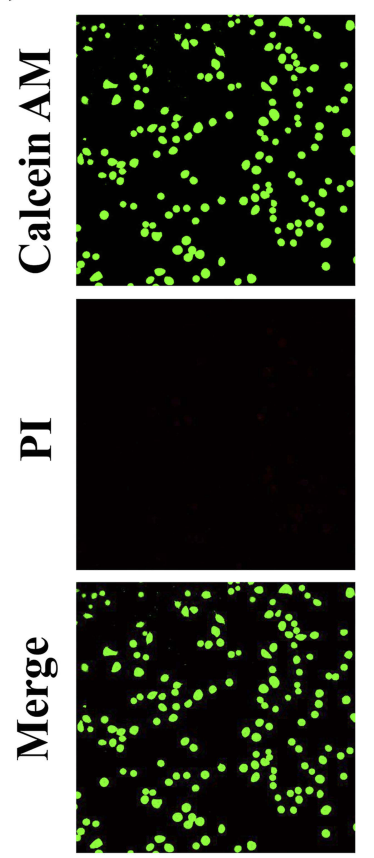

Control
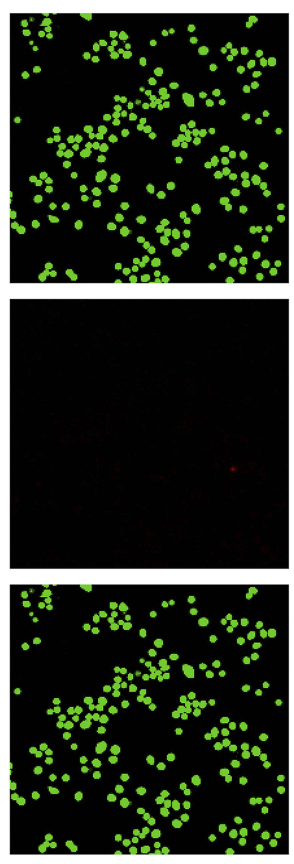

MPDA-PEG
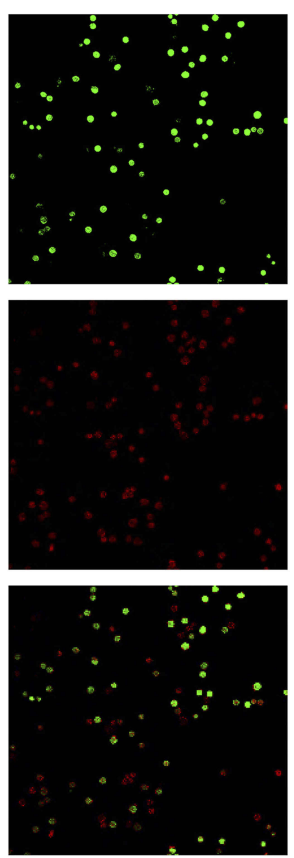

MPDA-PEG-PTX

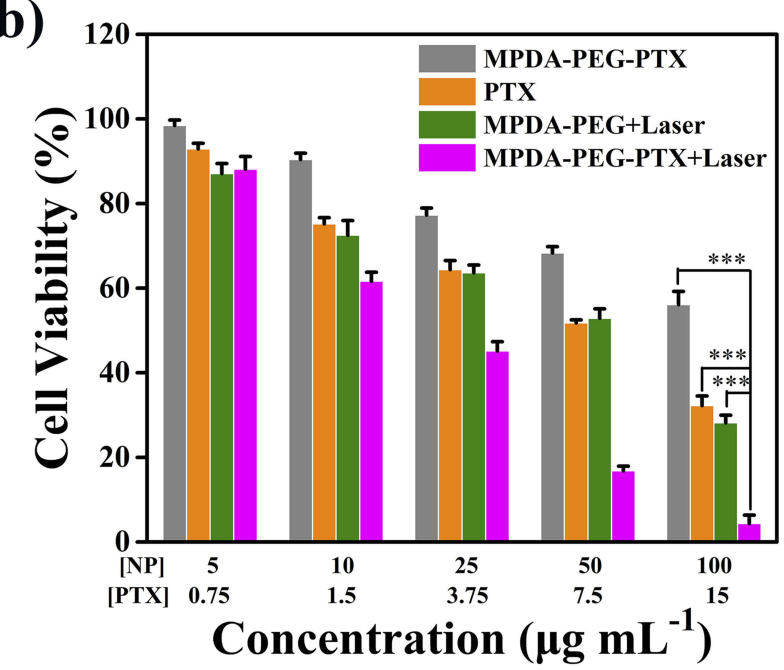

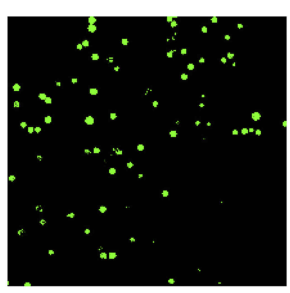
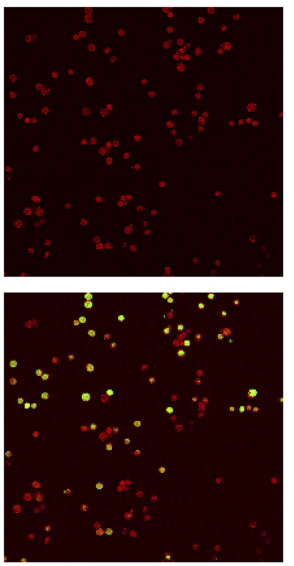

MPDA-PEG +Laser
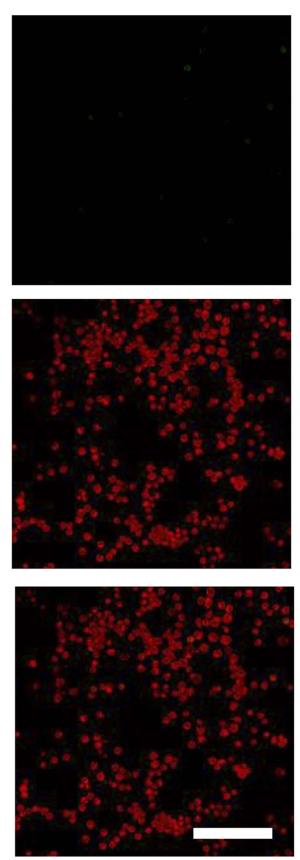

MPDA-PEG-PTX +Laser

Figure 5 (A) Cell viability of HEK-293T cells incubated with MPDA-PEG nanoparticles for 24 hrs with different concentrations. (B) In vitro cytotoxicity of MPDA-PEG, PTX, MPDA-PEG-PTX, MPDA-PEG + Laser, and MPDA-PEG-PTX + Laser at various concentrations. Data are means \pm sd. $\mathrm{N}=6$. (C) CLSM images of Calcein AM (green fluorescence, live cells) and Propidium iodide (PI, red fluorescence, dead cells) co-stained A549 cells incubated with PTX, MPDA-PEG, MPDA-PEG-PTX, MPDA-PEG + Laser, and MPDA-PEG-PTX + Laser $\left(100 \mu \mathrm{gL}^{-1}\right)$. The scale bar is $100 \mu \mathrm{m}$. *** $\mathrm{p}<0.001$.

Abbreviations: MPDA-PEG, polyethylene glycol-modified mesoporous polydopamine; PTX, paclitaxel; CLSM, confocal laser scanning microscope. 
MPDA-PEG + Laser group shows appreciable therapeutic effect, there are still more than $30 \%$ of cancer cells survived even at high concentration of $100 \mu \mathrm{g} \mathrm{mL}^{-1}$. In contrast, the cell viability of MPDA-PEG-PTX + Laser group decreases significantly. More than $80 \%$ of cancer cells are killed at particle concentration of $50 \mu \mathrm{g} \mathrm{mL}$, when the particle concentration increases to $100 \mu \mathrm{g} \mathrm{mL}^{-1}$, almost all the A549 cells are destroyed. In brief, MPDA-PEG-PTX + Laser irradiation possesses great synergistically therapeutic effect (combination index $=0.71$, calculated by Chou -Talalay method, Supplementary data). ${ }^{51,52}$

Subsequently, to verify that MPDA-PEG-PTX nanoparticles could be phagocytosed, cellular uptake experiments are performed by A549 cells incubated with Cy5 modified MPDA-PEG-PTX for $4 \mathrm{hrs}$. Red fluorescence signal from the Cy5 within the MPDA-PEG-PTX could be observed after 4-hr incubation (Figure S6), suggesting that MPDA-PEG-PTX could be efficiently uptake by A549 cells.

Meanwhile, CLSM image of calcein-AM (green fluorescence) and propodium iodide (PI, red fluorescence) costained A549 cells further confirmed the synergistic therapeutic effect of MPDA-PEG-PTX. ${ }^{53}$ As shown in Figure 5C, cells in control group and MPDA-PEG group exhibit intense green fluorescence, indicative of living cells, while cells in PTX group, MPDA-PEG-PTX group and MPDA-PEG + Laser group show partial green fluorescence, which indicative of insufficient killing effect. By contrast, maximum cell death is observed in MPDA-PEGPTX + Laser group. The results show that the cells are hardly alive treated with MPDA-PEG-PTX + Laser, which demonstrates MPDA-PEG-PTX + Laser has better synergistic therapeutic effect in vitro.

\section{In Vivo Chemo-Photothermal Therapy}

Due to the strong NIR absorption, MPDA-PEG also can be used as contrast agent in photoacoustic imaging (PAI), which can provide the local nanoparticle distribution in the tumor region. The PA signal of MPDA-PEG exhibits a linearity enhancement in vitro with increasing concentrations, which illustrated the excellent PA property of MPDA-PEG (Figure S7). With the guidance of PAI, MPDA-PEG-PTX suspensions $\left(100 \mu \mathrm{L}, 4 \mathrm{mg} \mathrm{mL}^{-1}\right)$ are injected into tumor-bearing mice, PA signals in tumor region are recorded at different time points $(0,1,2,4,6$, 12, $24 \mathrm{hrs}$ ). As shown in Figure 6A and B, the PA signal increased gradually within the first 4-hr post-injection at tumor site and then decreased, which indicated that the maximum nanoparticle enrichment in the tumor region is achieved at 4-hr post-injection. Subsequently, the photothermal effect of MPDA-PEG-PTX in tumor-bearing mice is further investigated. A549 tumor-bearing mice are randomly divided into four groups of 3 mice per group, mice are injected with $100 \mu \mathrm{L}$ of MPDA-PEG-PTX dispersion (4 mg mL ${ }^{-1}$ ) meanwhile control group treated with PBS. Tumors are completely exposed to $808 \mathrm{~nm}$ laser (2 W $\mathrm{cm}^{-2}$ ) for $5 \mathrm{mins}$ at 2-hr, 4-hr or 6-hr post-injection (each group corresponds to a time point). Infrared camera is used to monitor the change of tumor temperature. As shown in Figure 6C and D, upon irradiation for $5 \mathrm{mins}$, the tumor temperature of mice at 4-hr post-injection group rapidly increased from $30.1^{\circ} \mathrm{C}$ to $56.7^{\circ} \mathrm{C}\left(\Delta \mathrm{T}=26.6^{\circ} \mathrm{C}\right)$, while the groups at $2-\mathrm{hr}$ and 6 -hr post-injection can raise up to $52.4^{\circ} \mathrm{C}$ and $49.2^{\circ} \mathrm{C}$, respectively, which is consisted with PAI results. As a comparison, the tumor temperature of control group treated with PBS is found to increase no more than $8^{\circ} \mathrm{C}$ after laser irradiation.

To assess the circulation capability, tumor-bearing mice are intravenously injected with Cy5 modified MPDA-PEGPTX by measuring the fluorescent signal of nanoparticles in different organs and blood. The accumulation amount of MPDA-PEG-PTX in tumor region (Figure S8A) is calculated as $5.86 \pm 1.64$ and $3.29 \pm 1.04 \%$ ID per $g$, respectively, at 4-hr and 24-hr post-injection. Meanwhile, the blood levels of the MPDA-PEG-PTX reduced gradually with increase in time, there was $8.64 \pm 2.09 \% \mathrm{ID} / \mathrm{g}$ of MPDA-PEG-PTX nanoparticles remain in blood at 24-hr post-injection (Figure S8B).

Inspired by the excellent accumulation and in vitro antitumor effect of MPDA-PEG-PTX nanoparticles, in vivo treatment efficacy is further evaluated in mice. A549 tumor-bearing mice with tumor volume of $\sim 80 \mathrm{~mm}^{3}$ are randomly divided into six groups of 5 mice per group. After particle suspensions are injected by tail vein, tumors are completely exposed to $808 \mathrm{~nm}$ laser at 4hr post-injection. As shown in Figure 7A, the control group shows a rapid tumor growth. In comparison, the tumor growth inhibition values (TGI) of free PTX,

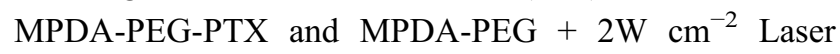
group (5 mins) are $35.2 \%, 44.5 \%$ and $56.3 \%$, which means the therapeutic effect of single chemotherapy or PTT alone is limited. Simultaneously, the most efficient treatment effect is observed when the mice are treated with

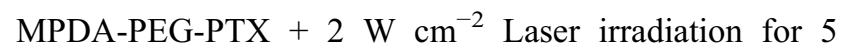
mins, tumor is completely ablated with no recrudescence, indicating that the synergistic effect of PTT and 
a)
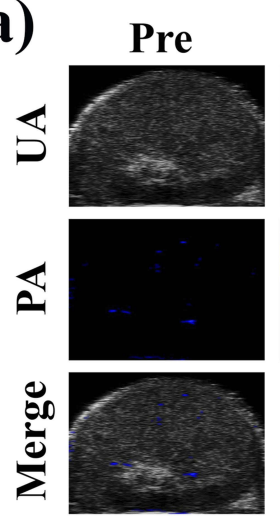

b)

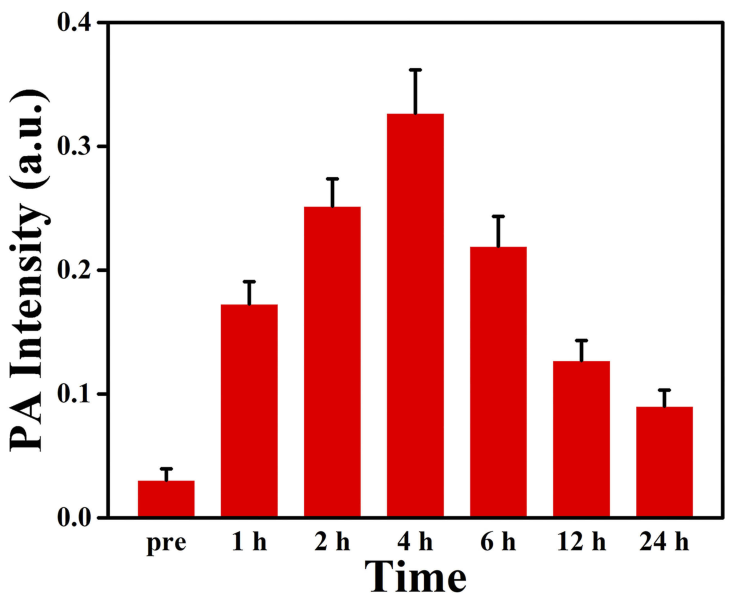

$4 \mathrm{~h}$
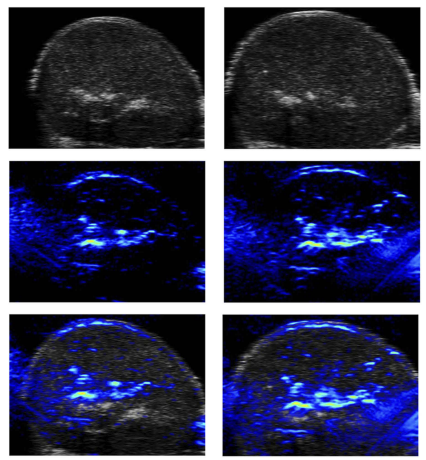

$6 \mathrm{~h}$
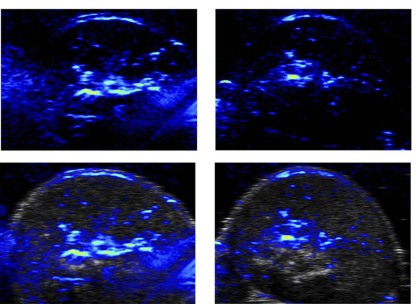

$12 \mathrm{~h}$
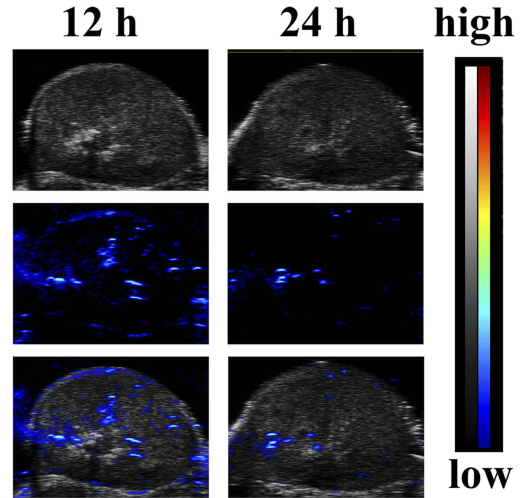

c)
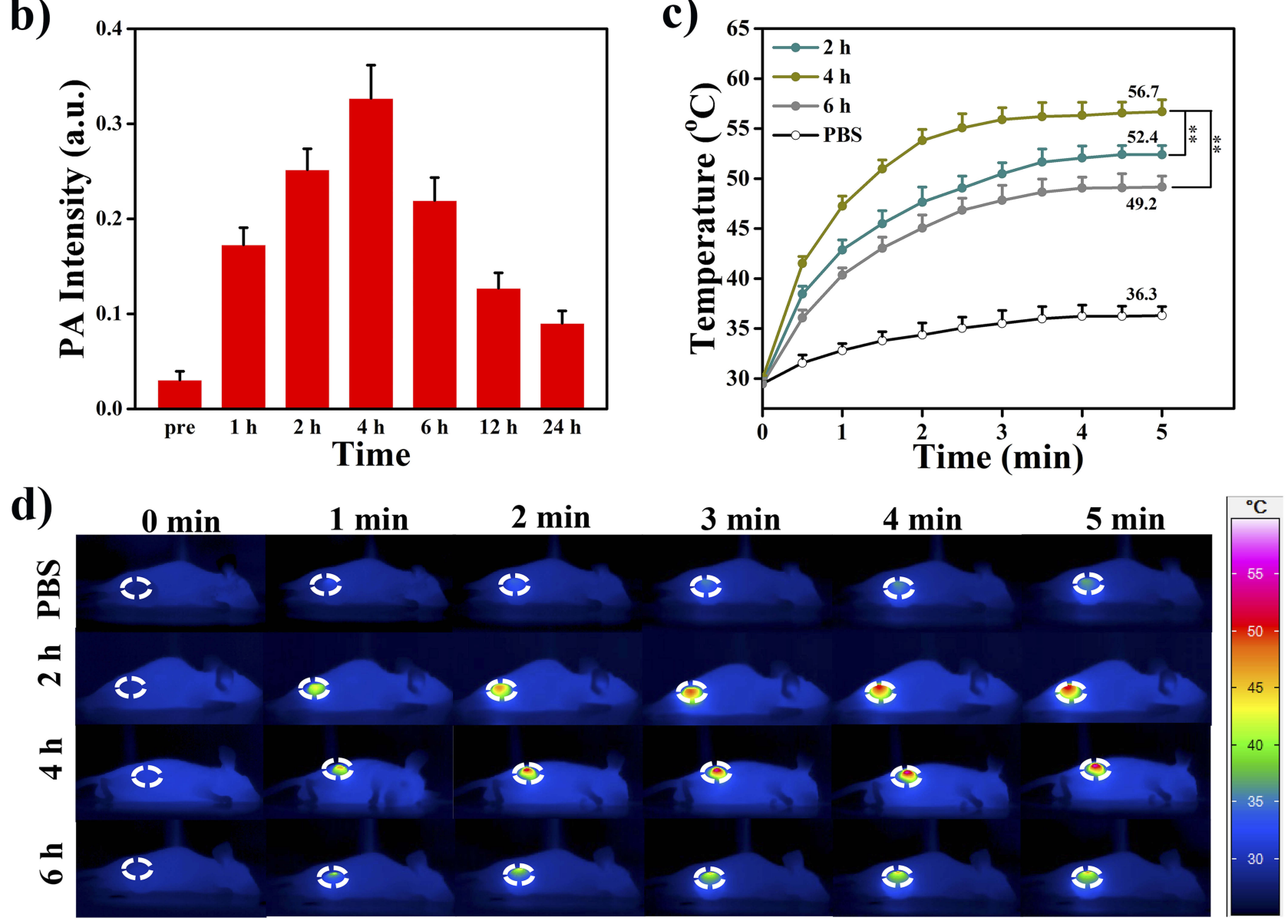

Figure 6 PA imaging $(\mathbf{A})$ and intensity $(\mathbf{B})$ in the tumor region after intravenously injected MPDA-PEG-PTX nanoparticles are recorded at different time points after intravenous administration. Local temperature (C) and thermal images (D) of four groups A549 tumor-bearing mice treated with PBS and MPDA-PEG-PTX (I $00 \mu \mathrm{L}, 4 \mathrm{mg} \mathrm{mL}^{-1}$ ) under NIR laser irradiation $\left(808 \mathrm{~nm}, 2 \mathrm{~W} \mathrm{~cm}^{-2}\right)$ for 5 mins at 2-hr, 4-hr, 6-hr post-injection. Data are means $\pm \mathrm{sd}$. $\mathrm{N}=3$. ${ }^{* *} \mathrm{p}<0.01$.

Abbreviations: PA, photoacoustic; MPDA-PEG-PTX, paclitaxel loaded polyethylene glycol-modified mesoporous polydopamine; PBS, phosphate buffer saline; NIR, near Infrared.

chemotherapy is effective. Moreover, a high antitumor capability (TGI, 93\%) is observed on the mice treated with MPDA-PEG-PTX $+1 \mathrm{~W} \mathrm{~cm}^{-2}$ Laser irradiation for 10 mins, suggesting the extraordinary synergistic therapeutic effect of MPDA-PEG-PTX could be realized at a lower power density. As shown in Figure 7C, the digital photographs of tumors on the 14th day after treatment shows that tumors in MPDA-PEG-PTX $+2 \mathrm{~W} \mathrm{~cm}^{-2}$ Laser group are completely ablated, while tumors in

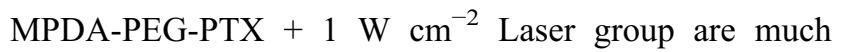
smaller than other groups. These results are found to agree fairly well with the tumor growth curves. 
a)

c)

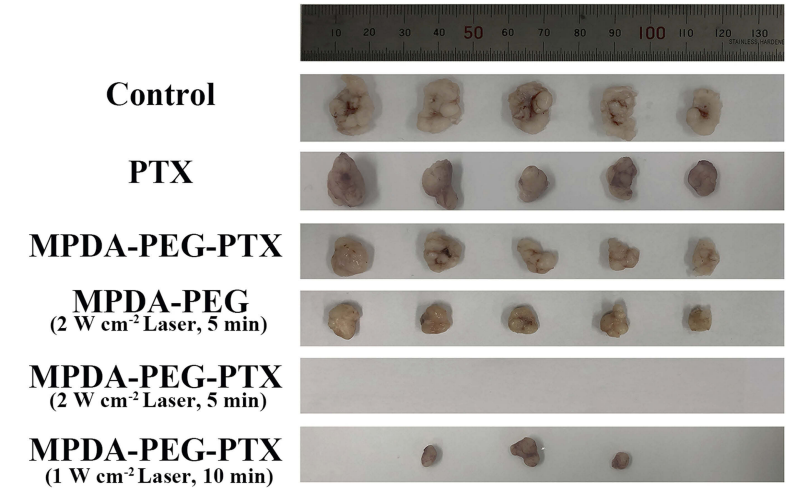

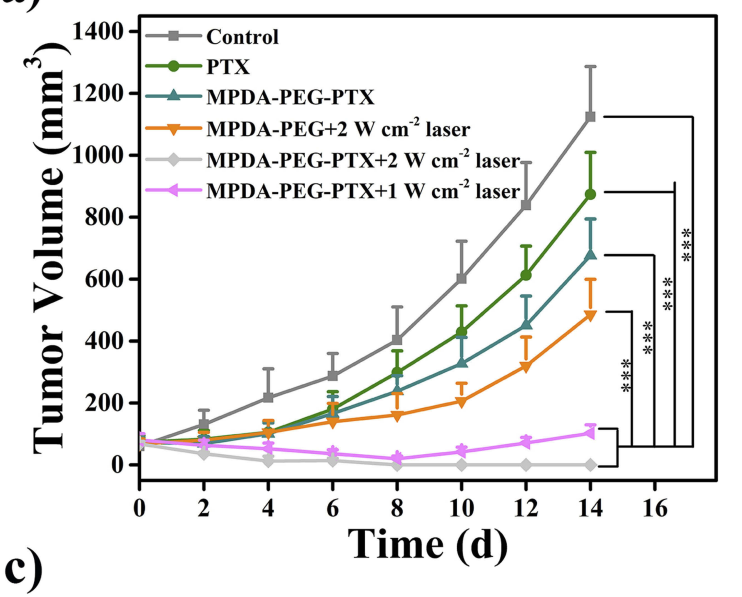

b)

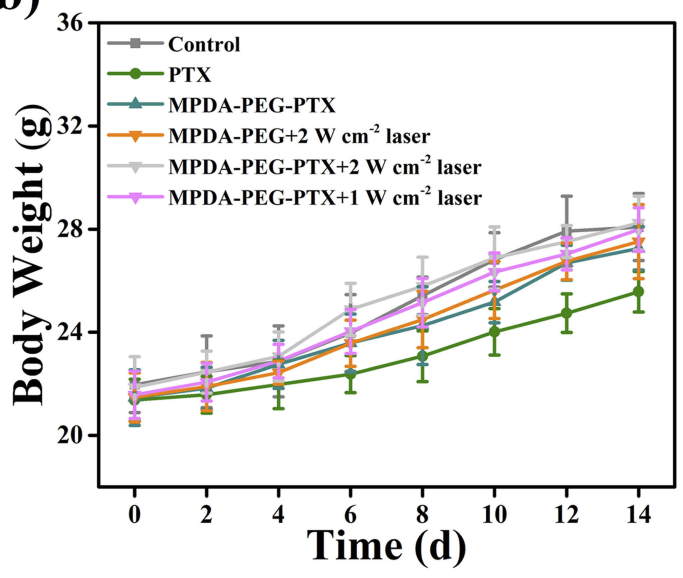

\section{Tumor Weight (g)}

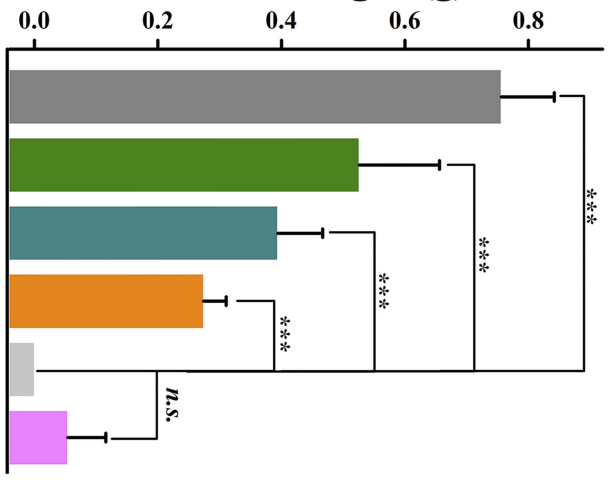

d)

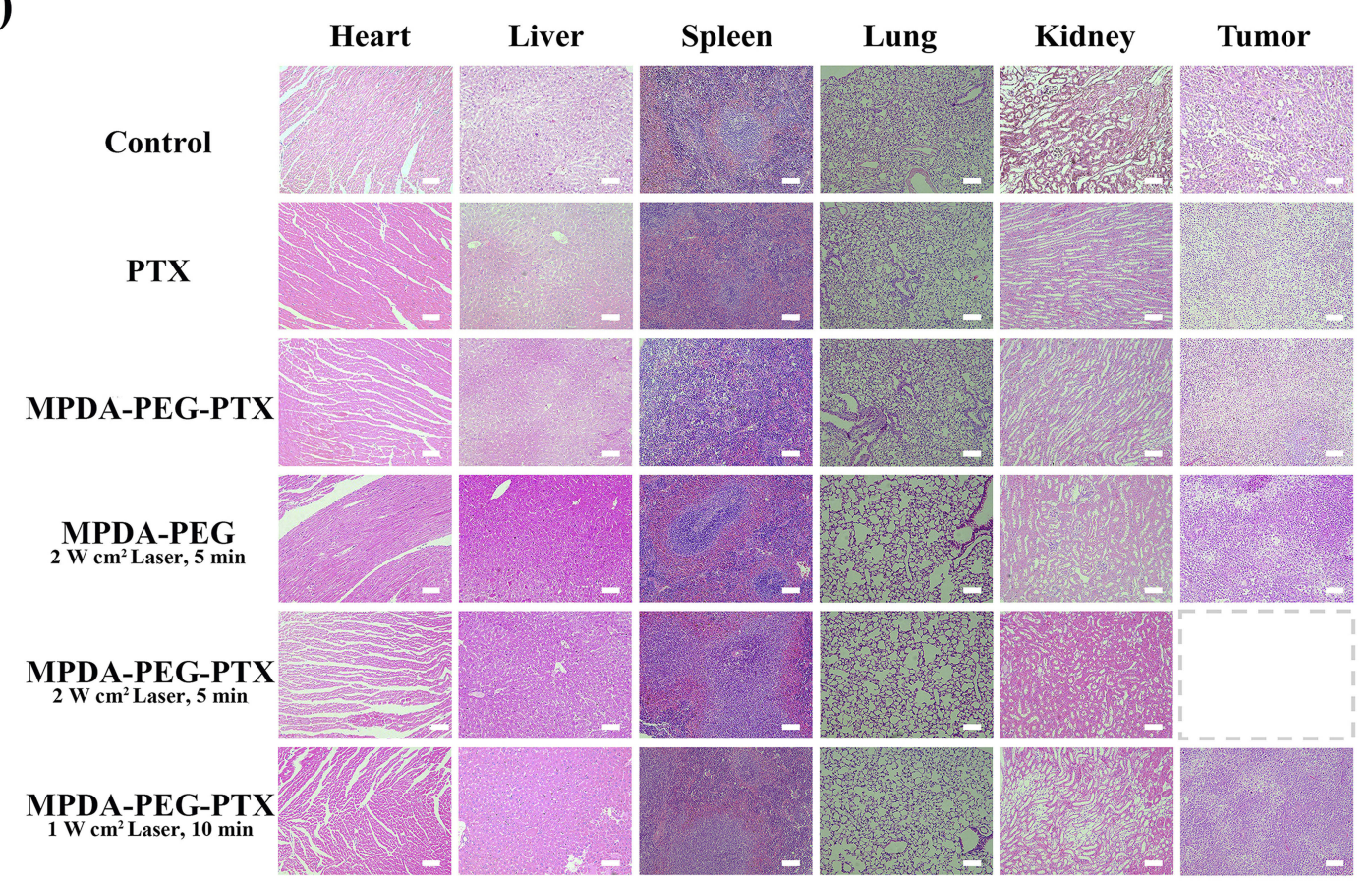

Figure 7 (A) Tumor volume curves of A549 tumor-bearing mice with different treatments (for laser, MPDA-PEG +2 W $\mathrm{cm}^{-2}$ Laser and MPDA-PEG-PTX $+2 \mathrm{~W} \mathrm{~cm}^{-2}$ Laser groups, tumors are irradiated by $808 \mathrm{~nm}$ laser for $5 \mathrm{mins}$, for MPDA-PEG-PTX $+\mathrm{I} \mathrm{W} \mathrm{cm}{ }^{-2}$ Laser group, tumors are irradiated by $808 \mathrm{~nm}$ laser for $10 \mathrm{mins}$ ). (B) Body weight curves of mice of each group. (C) Photos and weight of tumors dissected from each group on the I4th day after photothermal treatment. (D) H\&E staining images of major organs (heart, liver, spleen, lung, and kidney) and tumor tissues dissected from each group on the I4th day after photothermal treatment (40 X). The scale bars are I $00 \mu \mathrm{m}$. Data are means \pm sd. $N=5$. $* * * p<0.001$ and n.s. represents no significance.

Abbreviations: MPDA-PEG-PTX, paclitaxel loaded polyethylene glycol-modified mesoporous polydopamine; H\&E, hematoxylin-eosin staining. 
What is more, no apparent weight loss can be seen in all the groups of mice (Figure 7B), suggesting the MPDAPEG nanoparticles do not induce systemic toxicity. Subsequently, the in vivo biosafety of MPDA-PEG and MPDA-PEG-PTX nanoparticles is evaluated by blood biochemistry and haematology test. Haematology analysis revealed no significant differences in the blood parameters between nanoparticle-treated groups and control group at 48-hr post-injection (Table S3). Additionally, compared with control group, both the MPDA-PEG and MPDAPEG-PTX nanoparticles did not result in obvious increase in hepatic and renal indicator levels at 48 -hr post-injection (Figure S9), suggesting that the nanoparticle administration did not induce any acute hepatic or renal damage.

To further confirm the biocompatibility of MPDA-PEG nanoparticles, mice are dissected on the 14th day after treatment, and their tumors and major organs are subjected for histological analysis. As shown in Figure 7D, there are no obvious organ damage and inflammatory compared to PBS group, further verifying that the safety and low toxicity of MPDA-PEG-PTX nanoparticles. These results suggested that MPDA-PEG-PTX nanoparticles could serve as effective nanoplatform for cancer chemo-photothermal therapy in vivo with relatively low systemic toxicity.

\section{Conclusion}

In summary, we have successfully developed a PTXloaded MPDA-PEG nanoplatform for effective chemophotothermal synergistic therapy of tumor. MPDA-PEG nanoparticles with large pore volume and excellent biocompatibility are prepared by a modified emulsioninduced interface assembly strategy. With the increase of particle size, as-prepared MPDA-PEG nanoparticles not only possess an excellent photothermal conversion ability and enhanced photothermal effect, but also have a high payload capacity for PTX. Furthermore, the PTX release activity can be accelerated by elevated temperature under NIR irradiation. In vivo experiments further verified that MPDA-PEG-PTX nanoparticles can completely inhibit tumor growth with minimal side effect under NIR irradiation, exhibits better therapeutic effect than single chemotherapy and PTT. Our research proves that MPDA-PEG-PTX nanoparticles are expected to have wide applications in the synergetic chemo-photothermal therapy of cancer, and the introduction of biocompatible and biodegradable material could greatly decrease the adverse effect of chemotherapy in clinical treatment.

\section{Acknowledgments}

This work was financially supported by the National Key R\&D Program of China (Grant No. 2016YFC1100300) and the National Science Foundation of China (Grant Nos. 51873041 and 51473037).

\section{Disclosure}

The authors report no conflicts of interest in this work.

\section{References}

1. Ferrari M. Cancer nanotechnology: opportunities and challenges. Nat Rev Cancer. 2005;5(3):161-171. doi:10.1038/nrc1566

2. Shi J, Kantoff PW, Wooster R, Farokhzad OC. Cancer nanomedicine: progress, challenges and opportunities. Nat Rev Cancer. 2017;17 (1):20-37. doi:10.1038/nrc.2016.108

3. Haag R, Kratz F. Polymer therapeutics: concepts and applications. Angew Chem Int Ed. 2006;45(8):1198-1215. doi:10.1002/(ISSN)1521-3773

4. Wu MX, Yang YW. Metal-Organic Framework (MOF)-based drug/ cargo delivery and cancer therapy. Adv Mater. 2017;29(23):1606134. doi:10.1002/adma.201700681

5. Pan L, He Q, Liu J, et al. Nuclear-targeted drug delivery of TAT peptide-conjugated monodisperse mesoporous silica nanoparticles. $J$ Am Chem Soc. 2012;134(13):5722-5725. doi:10.1021/ja211035w

6. Zhong X, Yang K, Dong Z, et al. Polydopamine as a biocompatible multifunctional nanocarrier for combined radioisotope therapy and chemotherapy of cancer. Adv Funct Mater. 2015;25(47):7327-7336. doi:10.1002/adfm.201503587

7. Yang G, Xu L, Chao Y, et al. Hollow MnO2 as a tumor-microenvironment-responsive biodegradable nano-platform for combination therapy favoring antitumor immune responses. Nat Commun. 2017;8(1):902-915. doi:10.1038/s41467-017-01050-0

8. Xing Y, Zhang J, Chen F, Liu J, Cai K. Mesoporous polydopamine nanoparticles with co-delivery function for overcoming multidrug resistance via synergistic chemo-photothermal therapy. Nanoscale. 2017;9(25):8781-8790. doi:10.1039/c7nr01857f

9. Tian Y, Guo R, Yang W. Multifunctional nanotherapeutics for photothermal combination therapy of cancer. Adv Therap. 2018;1 (8):1800049. doi:10.1002/adtp.v1.8

10. Wang S, Huang P, Nie L, et al. Single continuous wave laser induced photodynamic/plasmonic photothermal therapy using photosensitizerfunctionalized gold nanostars. Adv Mater. 2013;25(22):3055-3061. doi:10.1002/adma.201204623

11. Chen D, Wang C, Nie X, et al. Photoacoustic imaging guided nearinfrared photothermal therapy using highly water-dispersible singlewalled carbon nanohorns as theranostic agents. Adv Funct Mater. 2014;24(42):6621-6628. doi:10.1002/adfm.201401560

12. Wang L, Sun Q, Wang X, et al. Using hollow carbon nanospheres as a light-induced free radical generator to overcome chemotherapy resistance. J Am Chem Soc. 2015;137(5):1947-1955. doi:10.1021/ja511560b

13. Sun H, Su J, Meng Q, et al. Cancer cell membrane-coated gold nanocages with hyperthermia-triggered drug release and homotypic target inhibit growth and metastasis of breast cancer. Adv Funct Mater. 2017;27(3):1604300. doi:10.1002/adfm.201604300

14. Wang D, Dong H, Li M, et al. Erythrocyte-cancer hybrid membrane camouflaged hollow copper sulfide nanoparticles for prolonged circulation life and homotypic-targeting photothermal/chemotherapy of melanoma. ACS Nano. 2018;12(6):5241-5252. doi:10.1021/acsnano.7b08355

15. Eyvazzadeh N, Shakeri-Zadeh A, Fekrazad R, Amini E, Ghaznavi H, Kamrava SK. Gold-coated magnetic nanoparticle as a nanotheranostic agent for magnetic resonance imaging and photothermal therapy of cancer. Lasers Med Sci. 2017;32(7):1469-1477. doi:10.1007/ s10103-017-2267-x 
16. Neshastehriz A, Tabei M, Maleki S, Eynali S, Shakeri-Zadeh A. Photothermal therapy using folate conjugated gold nanoparticles enhances the effects of $6 \mathrm{MV}$ X-ray on mouth epidermal carcinoma cells. J Photoch Photobio B. 2017;172:52-60. doi:10.1016/j. jphotobiol.2017.05.012

17. Ghaznavi H, Hosseini-Nami S, Kamrava SK, et al. Folic acid conjugated PEG coated gold-iron oxide core-shell nanocomplex as a potential agent for targeted photothermal therapy of cancer. Artif Cell Nanomed B. 2018;46(8):1594-1604. doi:10.1080/ 21691401.2017.1384384

18. Huang X, Ivan HE-S, Wei Q, El-Sayed MA. Cancer cell imaging and photothermal therapy in the near-infrared region by using gold nanorods. J Am Chem Soc. 2006;128(6):2115-2120. doi:10.1021/ja057254a

19. Shi X, Gong H, Li Y, Wang C, Cheng L, Liu Z. Graphene-based magnetic plasmonic nanocomposite for dual bioimaging and photothermal therapy. Biomaterials. 2013;34(20):4786-4793. doi:10.1016/ j.biomaterials.2013.03.023

20. Lynge ME, van der Westen R, Postma A, Stadler B. Polydopamine-a nature-inspired polymer coating for biomedical science. Nanoscale. 2011;3(12):4916-4928. doi:10.1039/c1nr10969c

21. Lee H, Dellatore SM, Miller WM, Messersmith PB. Mussel-inspired surface chemistry for multifunctional coatings. Science. 2007;318 (5849):426-430. doi:10.1126/science.1147241

22. Liu Y, Ai K, Lu L. Polydopamine and its derivative materials: synthesis and promising applications in energy, environmental, and biomedical fields. Chem Rev. 2014;114(9):5057-5115. doi:10.1021/ cr400407a

23. Liu X, Cao J, Li H, et al. Mussel-inspired polydopamine: a biocompatible and ultrastable coating for nanoparticles in vivo. ACS Nano. 2013;7(10):9384-9395. doi:10.1021/nn404117j

24. Jiang Q, Luo Z, Men Y, et al. Red blood cell membrane-camouflaged melanin nanoparticles for enhanced photothermal therapy. Biomaterials. 2017;143:29-45. doi:10.1016/j.biomaterials.2017.07.027

25. Liu Y, Ai K, Liu J, Deng M, He Y, Lu L. Dopamine-melanin colloidal nanospheres: an efficient near-infrared photothermal therapeutic agent for in vivo cancer therapy. Adv Mater. 2013;25(9):1353-1359. doi:10.1002/adma.201204683

26. Li WQ, Wang Z, Hao S, et al. Mitochondria-targeting polydopamine nanoparticles to deliver doxorubicin for overcoming drug resistance. ACS Appl Mater Interfaces. 2017;9(20):16793-16802. doi:10.1021/ acsami.7b01540

27. Cui J, Wang Y, Postma A, Hao J, Hosta-Rigau L, Caruso F. Monodisperse polymer capsules: tailoring size, shell thickness, and hydrophobic cargo loading via emulsion templating. Adv Funct Mater. 2010;20(10):1625-1631. doi:10.1002/adfm.v20:10

28. Guan BY, Yu L, Lou XW. Formation of asymmetric bowl-like mesoporous particles via emulsion-induced interface anisotropic assembly. $J \mathrm{Am}$ Chem Soc. 2016;138(35):11306-11311. doi:10.1021/jacs.6b06558

29. Ai K, Liu Y, Ruan C, Lu L, Lu GM. Sp2 C-dominant N-doped carbon sub-micrometer spheres with a tunable size: a versatile platform for highly efficient oxygen-reduction catalysts. Adv Mater. 2013;25 (7):998-1003. doi:10.1002/adma.201203923

30. Roper DK, Ahn W, Hoepfner M. Microscale heat transfer transduced by surface plasmon resonant gold nanoparticles. J Phys Chem C. 2007;111(9):3636-3641. doi:10.1021/jp064341w

31. Prencipe G, Tabakman SM, Welsher K, et al. PEG branched polymer for functionalization of nanomaterials with ultralong blood circulation. J Am Chem Soc. 2009;131:4783-4787. doi:10.1021/ja809086q

32. Park J, Brust TF, Lee HJ, Lee SC, Watts VJ, Yeo Y. Polydopamine-based simple and versatile surface modification of polymeric nano drug carriers. ACS Nano. 2014;8(4):3347-3356. doi: $10.1021 / \mathrm{nn} 405809 \mathrm{c}$

33. Awasthi VD, Garcia D, Goins BA, Phillips WT. Circulation and biodistribution profiles of long-circulating PEG-liposomes of various sizes in rabbits. Int J Pharm. 2003;253(1-2):121-132. doi:10.1016/ s0378-5173(02)00703-2
34. Ju KY, Lee Y, Lee S, Park SB, Lee JK. Bioinspired polymerization of dopamine to generate melanin-like nanoparticles having an excellent free-radical-scavenging property. Biomacromolecules. 2011;12 (3):625-632. doi:10.1021/bm101281b

35. Ghosh K, Kumar M, Maruyama T, Ando Y. Micro-structural, electron-spectroscopic and field-emission studies of carbon nitride nanotubes grown from cage-like and linear carbon sources. Carbon. 2009;47(6):1565-1575. doi:10.1016/j.carbon.2009.02.007

36. Ju KY, Lee S, Pyo J, Choo J, Lee JK. Bio-inspired development of a dual-mode nanoprobe for MRI and Raman imaging. Small. 2015;11 (1):84-89. doi:10.1002/smll.201401611

37. Zhou T, Zhou Y, Ma R, et al. Nitrogen-doped hollow mesoporous carbon spheres as a highly active and stable metal-free electrocatalyst for oxygen reduction. Carbon. 2017;114:177-186. doi:10.1016/j. carbon.2016.12.011

38. Guan BY, Zhang SL, Lou XW. Realization of walnut-shaped particles with macro-/mesoporous open channels through pore architecture manipulation and their use in electrocatalytic oxygen reduction. Angew Chem Int Ed. 2018;57(21):6176-6180. doi:10.1002/anie.201801876

39. Tian Y, Zhang J, Tang S, Zhou L, Yang W. Polypyrrole composite nanoparticles with morphology-dependent photothermal effect and immunological responses. Small. 2016;12(6):721-726. doi:10.1002/ smll.201503319

40. Yang P, Tian Y, Men Y, et al. Metal-organic frameworks-derived carbon nanoparticles for photoacoustic imaging-guided photothermal/photodynamic combined therapy. ACS Appl Mater Interfaces. 2018;10:42039-42049. doi:10.1021/acsami.8b15828

41. Cheng L, Wang C, Feng L, Yang K, Liu Z. Functional nanomaterials for phototherapies of cancer. Chem Rev. 2014;114(21):10869-10939. doi:10.1021/cr400532z

42. Duarte M-D, Pais-Silva C, Dias DR, Moreira AF, Correia IJ. Strategies to improve cancer photothermal therapy mediated by nanomaterials. Adv Healthcare Mater. 2017;6(10):1700073. doi:10.1002/ adhm.201700073

43. Cave AC, Brewer AC, Narayanapanicker A, et al. NADPH oxidases in cardiovascular health and disease. Antioxid Redox Signal. 2006;8 (5-6):691-728. doi:10.1089/ars.2006.8.691

44. Zhang P, Huang YX, Liu H, et al. A PEG-Fmoc conjugate as a nanocarrier for paclitaxel. Biomaterials. 2014;35(25):7146-7156. doi:10.1016/j.biomaterials.2014.04.108

45. Wu M, Xia XM, Cui C, et al. Highly efficient loading of amorphous paclitaxel in mesoporous hematite nanorods and their in vitro antitumor activity. J Mater Chem B. 2013;1(12):1687-1695. doi:10.1039/ c3tb00472d

46. Vivero-Escoto JL, Slowing II, Trewyn BG, Lin VS. Mesoporous silica nanoparticles for intracellular controlled drug delivery. Small. 2010;6(18):1952-1967. doi:10.1002/smll.200901789

47. Li X, Wang X, Sha L, et al. Thermosensitive lipid bilayer-coated mesoporous carbon nanoparticles for synergistic thermochemotherapy of tumor. ACS Appl Mater Interfaces. 2018;10(23):19386-19397. doi:10.1021/acsami.8b03008

48. Alamzadeh Z, Beik J, Mahabadi VP, et al. Ultrastructural and optical characteristics of cancer cells treated by a nanotechnology based chemo-photothermal therapy method. $J$ Photoch Photobio $B$. 2019;192:19-25. doi:10.1016/j.jphotobiol.2019.01.005

49. Beik J, Khateri M, Khosravi Z, et al. Gold nanoparticles in combinatorial cancer therapy strategies. Coord Chem Rev. 2019;387:299324. doi:10.1016/j.ccr.2019.02.025

50. Mirrahimi M, Abed Z, Beik J, et al. A thermo-responsive alginate nanogel platform co-loaded with gold nanoparticles and cisplatin for combined cancer chemo-photothermal therapy. Pharmacol Res. 2019;143:178-185. doi:10.1016/j.phrs.2019.01.005

51. Chou TC, Talalay P. Quantitative analysis of dose-effect relationships: the combined effects of multiple drugs or enzyme inhibitors. Adv Enzyme Regul. 1984;22:27-55. doi:10.1016/0065-2571(84) 90007-4 
52. Jiao Y, Sun Y, Tang X, Ren Q, Yang W. Tumor-targeting multifunctional rattle-type theranostic nanoparticles for MRI/NIRF bimodal imaging and delivery of hydrophobic drugs. Small. 2015;11 (16):1962-1974. doi:10.1002/smll.201402297
53. Shen S, Wang S, Zheng R, et al. Magnetic nanoparticle clusters for photothermal therapy with near-infrared irradiation. Biomaterials. 2015;39:67-74. doi:10.1016/j.biomaterials.2014.10.064

\section{Publish your work in this journal}

The International Journal of Nanomedicine is an international, peerreviewed journal focusing on the application of nanotechnology in diagnostics, therapeutics, and drug delivery systems throughout the biomedical field. This journal is indexed on PubMed Central, MedLine, CAS, SciSearch ${ }^{\mathbb{B}}$, Current Contents ${ }^{\mathbb{B}} /$ Clinical Medicine,
Journal Citation Reports/Science Edition, EMBase, Scopus and the Elsevier Bibliographic databases. The manuscript management system is completely online and includes a very quick and fair peer-review system, which is all easy to use. Visit http://www.dovepress.com/ testimonials.php to read real quotes from published authors. 